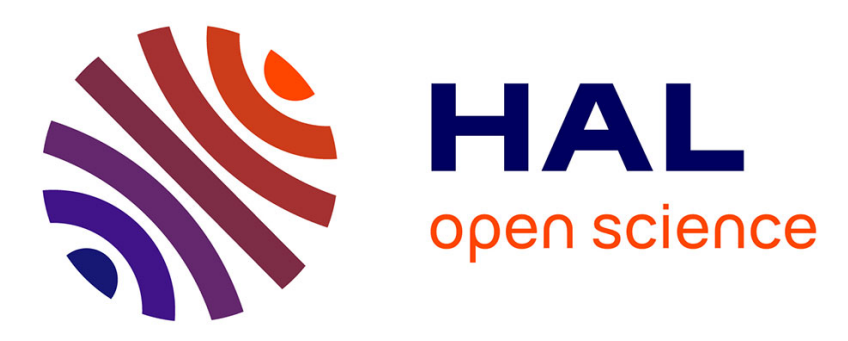

\title{
Optimal perturbation for two-dimensional vortex systems: route to non-axisymmetric state
}

Navrose Navrose, H. G Johnson, Vincent Brion, Laurent Jacquin, Jean-Christophe Robinet

\section{- To cite this version:}

Navrose Navrose, H. G Johnson, Vincent Brion, Laurent Jacquin, Jean-Christophe Robinet. Optimal perturbation for two-dimensional vortex systems: route to non-axisymmetric state. Journal of Fluid Mechanics, 2018, 855, pp.922-952. 10.1017/jfm.2018.689 hal-02906092

\section{HAL Id: hal-02906092 \\ https://hal.science/hal-02906092}

Submitted on 24 Jul 2020

HAL is a multi-disciplinary open access archive for the deposit and dissemination of scientific research documents, whether they are published or not. The documents may come from teaching and research institutions in France or abroad, or from public or private research centers.
L'archive ouverte pluridisciplinaire HAL, est destinée au dépôt et à la diffusion de documents scientifiques de niveau recherche, publiés ou non, émanant des établissements d'enseignement et de recherche français ou étrangers, des laboratoires publics ou privés. 


\title{
Optimal perturbation for $2 D$ vortex systems: route to non-axisymmetric state
}

\author{
Navrose $^{1},{ }^{3} \dagger$, H. G. Johnson ${ }^{1}$, V. Brion ${ }^{1}$, L. Jacquin ${ }^{1}$ and J. C. \\ Robinet $^{2}$ \\ ${ }^{1}$ Département Aérodynamique Aéroélasticité Aéroacoustique, ONERA, Meudon, France \\ ${ }^{2}$ DynFluid Laboratory, Arts et Métiers ParisTech, Paris, France \\ ${ }^{3}$ Department of Aerospace Engineering, Indian Institute of Technology Kanpur, UP, India
}

(Received $\mathrm{xx}$; revised $\mathrm{xx}$; accepted $\mathrm{xx}$ )

We investigate perturbations that maximize the gain of disturbance energy in twodimensional isolated vortex and counter-rotating vortex-pair. The optimization is carried out using the method of Lagrange multipliers. For low initial energy of the perturbation $(E(0))$, nonlinear optimal perturbation/gain is found to be the same as linear optimal perturbation/gain. Beyond a certain threshold $E(0)$, optimal perturbation/gain obtained from linear and nonlinear computation is different. There exists a range of $E(0)$ for which nonlinear optimal gain is higher than linear optimal gain. For isolated vortex, higher value of nonlinear optimal gain is attributed to interaction among different azimuthal components, that is otherwise absent in a linearized system. Spiral dislocations are found in nonlinear optimal perturbation at the radial location where the most dominant wavenumber changes. Long-time nonlinear evolution of linear and nonlinear optimal perturbation is studied. The evolution shows that after the initial increment of perturbation energy, the vortex attains a quasi-steady state where the mean perturbation energy decreases on a slow time-scale. The quasi-steady vortex state is non-axisymmetric and its shape depends on the initial perturbation. It is observed that the life of a quasi-steady vortex state obtained using nonlinear optimal perturbation is longer than that obtained using linear optimal perturbation. For counter-rotating vortex-pair, the mechanism that maximizes the energy gain is found to be similar to that of the isolated vortex. Within the linear framework optimal perturbation for a vortex pair can be either symmetric or anti-symmetric, whereas, the structure of the nonlinear optimal perturbation, beyond the threshold $E(0)$, is always asymmetric. Quasi-steady state for counter-rotating vortex pair is not observed.

Key words:

\section{Introduction}

From giant cyclones to small-scale structures in turbulence, vortices are present in almost all natural flows. They play a crucial role in the transport of mass, momentum and energy in flow processes. Perhaps this is why Küchemann (1965) observed that "vortices are the sinews and muscles of fluid motion". As such, vortices have been extensively studied for their role in flow transition and turbulence. For a detailed examination on

$\dagger$ Email address for correspondence: navrose@iitk.ac.in 
different types of vortical flows, the interested reader is referred to Lugt (1983), Saffman (1992) and Green (1995).

The mathematical analysis of vortex systems can be traced back as early as 1880 . Kelvin (1880) investigated wave motions in a column of uniform vorticity surrounded by irrotational flow (popularly referred to as the Rankine vortex model). These so-called Kelvin waves were the starting point for several later works on the dynamics of Rankine vortex as well as other vortical flows. One such topic is stability of vortex columns. A general perturbation to Rankine vortex can be represented as a linear combination of the various Kelvin waves. If the perturbation is small, nonlinear term in the flow equations can be dropped. Within the linear framework, the evolution of each wave component occurs independently from the rest. Therefore the stability of a vortex column can be determined by analyzing the stability of each wave component separately. For inviscid vortex, the waves are neutrally stable. In the presence of viscosity the waves decay exponentially in time. For other vortex models (for example the Lamb-Oseen vortex), additional waves can exist (Fabre, Sipp \& Jacquin 2006). Still, all waves decay exponentially under the action of viscosity. Thus, any infinitesimal perturbation to an isolated vortex will eventually decay in time.

Linear Stability Analysis (LSA) is useful for studying the asymptotic response of a fluid system to infinitesimal perturbations. Long-term, the least stable - or most unstable mode is expected to dominate the flow evolution. However, many flow processes occur on a finite time scale. LSA fails to capture such transient processes that might contribute to the growth of perturbation energy $(E)$. The transient growth of perturbation energy is attributed to non-normality of the linearized Navier-Stokes operator (Farrell 1988; Trefethen et al. 1993). As a consequence of non-normality, the eigenmodes associated with the operator are non-orthogonal. Therefore, it is possible to combine different eigenmodes and form an initial perturbation for which the rate of change of perturbation energy $(d E / d t)$ with time is positive, despite all the eigenmodes being linearly stable. For unstable systems, eigenmodes can be suitably combined to give a higher growth rate of perturbation energy than the most unstable eigenmode. During the transient phase (of stable/unstable flows), the perturbation may become strong enough for secondary instabilities to occur. The subsequent flow evolution will then be different from that of the linearized system. An example for such behavior is the plane Couette flow (PCF). LSA predicts that the PCF is stable for all Reynolds numbers $(R e)$. However, experiments show that turbulence can be produced and sustained in the PCF for $R e$ as low as $\sim 300$ (Barkley 2016). The transient growth of perturbation energy might fill-in the gap between experimental observation and results of LSA.

The inability of LSA to describe flow evolution during the transient phase motivates us to take an alternate perspective on stability of flow systems. The new approach is based on seeking perturbation that maximizes the perturbation energy over a given horizon time $(T)$. We note that this approach introduces $T$ as a stability parameter. The perturbation satisfying the maximization criterion is referred to as the optimal perturbation and the corresponding energy gain at $t=T$ is the optimal gain. The shape of the optimal perturbation and the value of optimal gain depends on $T$. A recent article by Schmid \& Brandt (2014) provides an overview of the various tools and techniques that have been developed to compute optimal gain and perturbations in flow systems.

Optimal perturbation for an isolated vortex has been studied mainly within the linear framework (Antkowiak \& Brancher 2004, 2007; Pradeep \& Hussain 2006; Mao \& Sherwin 2011, 2012). Antkowiak \& Brancher (2004) carried out optimal perturbation analysis for two-dimensional $(2 D)$ and three-dimensional $(3 D)$ perturbations with azimuthal wavenumber $m=1$. They observed that linear optimal perturbation consists of vorticity 
filaments in a spiral arrangement near the outer periphery of the vortex core. The energy growth mechanism was attributed to a combination of the Orr mechanism (Orr 1907) and vortex induction. During Orr mechanism the spiral filaments that are inclined against the base flow is uncoiled resulting in the growth of perturbation energy. This uncoiling, in-turn, was found to promote vortex induction along the vortex axis. Furthermore they reported that linear optimal gain for small axial wavenumbers $(k \rightarrow 0)$ is significantly higher than for large wavenumbers. For $k=0$, linear optimal gain was found to increase linearly with $T$. Pradeep \& Hussain (2006) investigated $3 D$ linear optimal perturbation for various $(m, k)$ combinations and identified the corresponding physical mechanism behind the energy growth. They reported that the axisymmetric mode $(m=0)$ results in the largest growth of perturbation energy in the full computational domain. The $m=1$ (bending) mode, on the other hand, causes the largest perturbation to the vortex core. They speculated that the bending wave might be responsible for core transition to turbulence. Antkowiak \& Brancher (2007) observed that a perturbation consisting of a stack of azimuthal velocity streaks leads to an amplification of perturbation energy in columnar vortices. The velocity stack evolves to form vortex rings around the core of the vortex. Based on this observation they surmised that a similar mechanism might be at play in the development of vortex rings around a columnar vortex when it is submerged in a turbulent background.

In the present work, we investigate nonlinear optimal perturbation of an isolated LambOseen vortex. The only work in this direction is by Bisanti (2013). Bisanti's analysis was restricted to $m=2$ perturbations, and the value of nonlinear optimal gain obtained in the work was found to be lower than the linear optimal gain. We do not put any restriction with respect to $m$ in our work. Our results show that the interaction between different azimuthal components can result in higher optimal gain than linear optimal gain. Bisanti (2013) reported that if the initial energy of the perturbation is large, the $m=2$ nonlinear optimal perturbation triggers a subcritical bifurcation to a quasi-steady rotating tripolar perturbation. The existence of quasi-steady non-axisymmetric vortex states has been established in several earlier works (for example, Rossi, Lingevitch \& Bernoff (1997) and Le Dizès (2000)). We have studied the long-term nonlinear evolution of the linear and nonlinear optimal perturbations. Dipolar, tripolar and quadrupolar vortex states that retain their shape for several rotation periods are observed in our simulations. This suggests that optimal perturbation serves as a good initial condition to realize quasi-steady non-axisymmetric vortex states. Our work also brings out the significance of critical layer in the evolution of non-axisymmetric vortices.

One of the major motivations for studying vortex dynamics is its application in the analysis of aircraft wakes. Vorticity sheet generated over the wings of the aircraft roll-up downstream to form a pair of counter-rotating vortices. These wake vortices are quite resilient to surrounding turbulence and remain in the atmosphere for many rotation periods of the vortex. If an aircraft encounters wake vortices, there is a possibility of loss of control. Such a scenario would be catastrophic during take-off or landing as the aircraft may not have sufficient altitude to recover. To avoid this situation, regulations have been imposed to set minimum distance between two aircrafts. This allows time for the wake vortices to decay naturally or to be convected away. Due to the continuing increase in demand for air transport, such regulations have led to the saturation of aircraft operations in many major airports. There is an urgent need to find solution to the saturation problem. One possibility would be to develop strategies for alleviation of wake vortices. In this regard, optimal perturbation in double vortex systems may provide a lead. It is expected that the optimal perturbation will hasten the transition to turbulence in wake vortices, thereby rendering the wake benign for vortex encounter. 
Compared with the single vortex system, the double vortex system has been considerably less explored. The stability of a vortex pair was investigated in earlier works (for example Crow 1970; Tsai \& Widnall 1976; Pierrehumbert 1980). In 2D, Brion, Sipp \& Jacquin (2014) showed the existence of several unstable modes for a vortex-pair. Recently, Jugier (2016) pursued this flow and investigated linear optimal perturbation for a pair of counter-rotating vortices. He found that a transient mechanism can lead to higher energy gain than the most unstable mode. In the present work we investigate linear and nonlinear optimal perturbation in a counter-rotating vortex pair. The vortex-pair is obtained by superimposing velocity field for two Lamb-Oseen vortices of opposite circulation placed at a certain distance from each other, and letting the resultant flow to evolve for some time. The flow evolution stage is important as the superimposed flow is not an exact solution of Navier-Stokes equations. Such technique has been used in earlier numerical works on double vortex systems (Brion 2009; Jugier 2016). Our results show that the mechanism of transient energy growth in counter-rotating vortex pair is similar to that of isolated vortex. This reinforces the relevance of studying an isolated vortex to the analysis of vortex pair dynamics. However, unlike an isolated vortex, nonlinear transient processes does not lead to a quasi-steady dynamics for the vortex pair. Instead, the flow switches to an unstable evolution beyond a certain time. In this situation, optimal perturbation (linear/nonlinear) can be used to hasten the rate of flow destabilization.

The paper has been organized as follows. Section 2 describes the governing equations and the optimization strategy. Next, results from optimal analysis for an isolated LambOseen vortex are presented in Section 3. Results for counter-rotating vortex pair are presented in Section 4. We conclude the paper in Section 5.

\section{Problem formulation}

\subsection{Governing equations}

The flow is governed by the incompressible Navier-Stokes equations:

$$
\begin{aligned}
\nabla \cdot \boldsymbol{u} & =0 \\
\frac{\partial \boldsymbol{u}}{\partial t}+(\boldsymbol{u} \cdot \nabla) \boldsymbol{u} & =-\nabla p+\frac{1}{R e} \nabla^{2} \boldsymbol{u},
\end{aligned}
$$

where $\boldsymbol{u}, p$ and Re are the velocity, pressure and Reynolds number, respectively. Equations (2.1)-(2.2) are accompanied by initial and boundary conditions for the flow variables. The flow (represented by $\boldsymbol{q}=(\boldsymbol{u}, p))$ is written as sum of base flow $(\boldsymbol{Q}=(\boldsymbol{U}, P))$ and perturbation $\left(\boldsymbol{q}^{\prime}=\left(\boldsymbol{u}^{\prime}, p^{\prime}\right)\right)$,

$$
\boldsymbol{q}=\boldsymbol{Q}+\boldsymbol{q}^{\prime}
$$

The decomposition (2.3) is generally useful when the base state evolves on a time scale that is much slower than the evolution of the perturbation. More often than not, the steady solution of equations (2.1)-(2.2) is selected as the base flow. Substituting (2.3) in equations (2.1)-(2.2) and subtracting from it the equations for the base state, we get the equations governing the evolution of the perturbation:

$$
\begin{aligned}
\nabla \cdot \boldsymbol{u}^{\prime} & =0, \\
\frac{\partial \boldsymbol{u}^{\prime}}{\partial t}+(\boldsymbol{U} \cdot \nabla) \boldsymbol{u}^{\prime}+\left(\boldsymbol{u}^{\prime} \cdot \nabla\right) \boldsymbol{U}+\left(\boldsymbol{u}^{\prime} \cdot \nabla\right) \boldsymbol{u}^{\prime} & =-\nabla p^{\prime}+\frac{1}{R e} \nabla^{2} \boldsymbol{u}^{\prime} .
\end{aligned}
$$

Equations (2.4)-(2.5) are accompanied by initial conditions for the perturbation $\left(\boldsymbol{q}_{t=0}^{\prime}\right)$ and homogeneous boundary conditions. In the linear framework, the nonlinear term $\left(\boldsymbol{u}^{\prime} \cdot \nabla\right) \boldsymbol{u}^{\prime}$ is dropped from equation (2.5). 


\subsection{Optimization}

We seek initial perturbation $\left(\boldsymbol{q}_{0}^{\prime}\right)$ that maximizes the gain of perturbation kinetic energy over a given horizon time $T$. The kinetic energy associated with the perturbation at any time instant is taken as

$$
E(t)=\int_{\Omega} \boldsymbol{u}^{\prime}(t) \cdot \boldsymbol{u}^{\prime}(t) d \Omega .
$$

where $\Omega$ is the computational domain. The energy gain with respect to the initial perturbation is

$$
G\left(\boldsymbol{q}_{0}^{\prime}, t\right)=E(t) / E(0)
$$

where $E(0)$ is the energy of the perturbation at $t=0$. Therefore optimal perturbation maximizes the value of $G$ at $T$. We use the method of Lagrange multipliers to solve the optimization problem. The Lagrangian functional, $\mathcal{L}$, is defined as

$$
\mathcal{L}\left(\boldsymbol{q}^{\prime}, \boldsymbol{q}^{+}, \boldsymbol{q}_{0}^{\prime}, \boldsymbol{q}_{0}^{+}, \lambda\right)=G\left(\boldsymbol{q}_{0}^{\prime} ; T\right)-\left\langle\boldsymbol{q}^{+}, \mathbf{F}\left(\boldsymbol{q}^{\prime}\right)\right\rangle-\lambda\left(E_{0}-E(0)\right),
$$

where $\mathbf{F}\left(\boldsymbol{q}^{\prime}\right)=0$ represents the equation system (2.4)-(2.5) (or the linearized version of it for linear optimization), $\boldsymbol{q}^{+}$and $\lambda$ are the Lagrange multipliers and, $\langle.,$.$\rangle denotes$ the inner product of two vectors,

$$
\langle\boldsymbol{a}, \boldsymbol{b}\rangle=\int_{T} \int_{\Omega} \boldsymbol{a} \cdot \boldsymbol{b} d \Omega d t .
$$

$\mathbf{F}$ is usually referred to as the Navier-Stokes operator. The boundary conditions accompanying equations (2.4)-(2.5) are implicit in the definition of $\mathcal{L} . \quad E_{0}$ is a parameter that constrains the kinetic energy of the perturbation at $t=0$ to a fixed value (equal to $\left.E_{0}\right)$ via the last term in the right hand side of equation (2.8). The constraint is set using a geometric update technique described later in this section. The optimization procedure, therefore, yields optimal perturbation/gain correponding to a given value of $E_{0}$. Within the linear framework, optimal gain is independent of the initial energy of the perturbation. Non-linear optimal gain/perturbation, on the other hand, depends on the value of $E_{0}$.

For optimality, the gradient of $\mathcal{L}$ with respect to all the variables should be zero. Setting to zero the gradient of $\mathcal{L}$ with respect to

(a) $\boldsymbol{q}^{+}$, returns equations (2.4)-(2.5),

(b) $\boldsymbol{q}^{\prime}$, gives the adjoint equations

$$
\begin{aligned}
\nabla \cdot \boldsymbol{u}^{+} & =0 \\
\frac{\partial \boldsymbol{u}^{+}}{\partial t}+(\boldsymbol{U} \cdot \nabla) \boldsymbol{u}^{+}+\left(\boldsymbol{u}^{\prime} \cdot \nabla\right) \boldsymbol{u}^{+} & =\boldsymbol{u}^{+} \cdot(\nabla \boldsymbol{U})^{T}+\boldsymbol{u}^{+} \cdot\left(\nabla \boldsymbol{u}^{\prime}\right)^{T} \\
& -\nabla p^{+}-\frac{1}{R e} \nabla^{2} \boldsymbol{u}^{+}
\end{aligned}
$$

(c) $\boldsymbol{q}_{0}^{+}$, gives the compatibility equation

$$
\boldsymbol{u}^{+}(T)=2 \frac{\boldsymbol{u}^{\prime}(T)}{E_{0}},
$$

(d) $\boldsymbol{q}_{0}^{\prime}$, gives the optimality equation

$$
\nabla_{\boldsymbol{q}_{0}^{\prime}} \mathcal{L}=\boldsymbol{u}^{+}(0)-2 \frac{E_{T}}{E_{0}^{2}} \boldsymbol{u}^{\prime}(0) .
$$


For a detailed derivation of equations (2.10)-(2.13) the interested reader is referred to Farrell (1988); Corbett \& Bottaro (2000); Zuccher, Bottaro \& Luchini (2006); Cherubini et al. (2011, 2013), Schmid \& Brandt (2014) and Kerswell (2018).

An iterative procedure is employed to arrive at the optimal initial condition. Each iteration loop consists of the following four steps:

1) equations (2.4)-(2.5) (also referred to as direct equations) are marched forward in time from $t=0$ to $T$,

2) equation (2.12) is used to compute the adjoint field at $T$,

3 ) equations (2.10)-(2.11) are solved backwards in time from $t=T$ to $t=0$,

4) equation (2.13) along with the geometric update technique proposed by Douglas, Amari \& Kung (2000) are used to set the next guess for $\boldsymbol{q}_{0}^{\prime}$.

The geometric update technique sets the initial energy of the perturbation. First, the component of $\nabla_{\boldsymbol{q}_{0}^{\prime}} \mathcal{L}$ that is normal to $\boldsymbol{q}_{0}^{\prime}$ is scaled as per the initial energy constraint,

$$
\boldsymbol{N}^{j}=E(0)^{1 / 2} \frac{\nabla_{\boldsymbol{q}_{0}^{\prime}} \mathcal{L}_{\perp}}{\left\|\nabla_{\boldsymbol{q}_{0}^{\prime}} \mathcal{L}_{\perp}\right\|},
$$

where $j$ is the iteration number and $\perp$ denotes normal component. The initial perturbation for step 1) of next iteration is then given by:

$$
\boldsymbol{q}_{0}^{\prime j+1}=\boldsymbol{q}_{0}^{\prime j} \cos (\alpha)+\boldsymbol{N}^{j} \sin (\alpha) .
$$

In equation (2.15) $\alpha$ is the step length and its starting value is unity. A simplified line search is implemented to choose the right value of $\alpha$. If at the end of step 1 the energy gain has higher value than the previous iteration, then $\alpha$ is not changed. If the gain is lower than the previous gain, $\alpha$ is reduced successively by a factor of two until the energy gain has a higher value compared to last iteration. For the first iteration loop, the initial perturbation for step 1 is random white noise. In linear optimization the terms $\left(\boldsymbol{u}^{\prime} \cdot \nabla\right) \boldsymbol{u}^{+}$and $\boldsymbol{u}^{+} \cdot\left(\nabla \boldsymbol{u}^{\prime}\right)^{T}$ are are not present in equation $(2.11)$.

The computations have been carried out using Nek5000 (Fischer, Lottes \& Kerkemeier 2008), which is an open source spectral element solver for incompressible and weakly compressible flows. For the single vortex system, a circular computational domain is used. For the double vortex system, computations are carried out in a rectangular domain. The computational domain is divided into a large number of spectral elements. Each spectral element is further discretized using Gauss-Lobatto-Legendre (GLL) points. Near the vortex core region, the spatial resolution of the mesh is kept high in order to capture the flow gradients accurately. The resolution decreases towards the boundary of the computational domain. Convergence studies have been carried out to establish the adequacy of the extent of the computational domain and mesh resolution. The effect of the size of the computational domain for an isolated vortex is discussed in Section 3.2.2.

\subsection{Linear Stability Analysis of Lamb-Oseen vortex}

The flow state is written in normal mode representation,

$$
\mathbf{q}^{\prime}(r, \theta, t)=\hat{\mathbf{q}}(r) e^{i m \theta} e^{\lambda t}
$$

where $m$ is the azimuthal wavenumber and $\hat{\mathbf{q}}$ and $\lambda$ are complex quantities. Substituting (2.16) in the linearized version of equations (2.4)-(2.5) we get a generalized eigenvalue problem:

$$
\mathbf{A} \hat{\mathbf{q}}=\lambda \mathbf{B} \hat{\mathbf{q}}
$$

The real and imaginary parts of $\lambda$ correspond to the growth rate and frequency of 
the eigenmode respectively. In the discrete version of the problem, $\mathbf{A}$ and $\mathbf{B}$ are nonsymmetric matrices. A shift-inverse transformation is used to compute the eigenmodes and eigenvalues.

\section{Isolated vortex}

\subsection{Base flow}

The base flow for carrying out the optimal analysis is a Lamb-Oseen vortex. Its velocity distribution in cylindrical coordinate system is given by:

$$
u_{r}=0, u_{\theta}=\frac{\Gamma}{2 \pi r}\left(1-e^{-r^{2} / a^{2}}\right)
$$

where $u_{r}$ and $u_{\theta}$ are the radial and azimuthal components of velocity, $\Gamma$ is the circulation associated with the Lamb-Oseen vortex, $a$ is the vortex dispersion radius and $r$ is the distance from the origin. The vortex strength $(\Gamma)$ is related to vorticity $(\omega)$ by:

$$
\Gamma=\int_{\Omega} \omega d \Omega
$$

In equation (3.2), the integration is carried out over the entire computational domain. The vortex dispersion radius $(a)$ is given by:

$$
a=\sqrt{\frac{\int_{\Omega} r^{2} \omega d \Omega}{\Gamma}} .
$$

The Lamb-Oseen vortex is assumed to be frozen in time. The length and velocity scales have been rendered non-dimensional using $a$ and $\Gamma / 2 \pi a$ respectively. The Reynolds number $(R e=\Gamma / 2 \pi \nu)$ for all simulations is $R e=5000$. Bernoff \& Lingevitch (1994) analyzed the relaxation of a perturbed Gaussian vortex and found that the perturbations evolve on a $R e^{1 / 3}$ time scale. For $R e=5000$, the time scale for evolution of perturbation is several orders lower than viscous dissipation $\left(\tau_{\nu}=2 \pi a^{2} / \nu\right)$. It is, therefore, reasonable to assume that the base flow is steady during perturbation evolution. This is confirmed in Appendix A where optimization results obtained for $T=4.8$ using an evolving base flow are found to be nearly the same as those obtained using the frozen base flow approach. For very large horizon times the frozen base flow approach is inadequate and serves as an approximation. Compared to an evolving base flow, the frozen base flow approach has significant advantages in terms of computational memory and time.

\subsection{Linear analysis}

\subsubsection{Optimal gain and perturbation}

Optimal perturbation for an isolated vortex has been studied mostly in $3 D$ and almost exclusively within the linear framework (Antkowiak \& Brancher 2004, 2007; Pradeep \& Hussain 2006). Antkowiak \& Brancher (2004) investigated linear optimal perturbation (LO-P) for azimuthal wavenumber $m=1$. In the present study, we extend the $2 D$-optimization work of Antkowiak \& Brancher (2004) by considering additional wavenumbers.

A general perturbed state of Lamb-Oseen vortex is amenable to Fourier decomposition in the azimuthal direction:

$$
\mathbf{q}^{\prime}(r, \theta, t)=\sum_{m \in \mathbb{Z}} \mathbf{q}_{m}(r, t) e^{i m \theta}+c . c .
$$




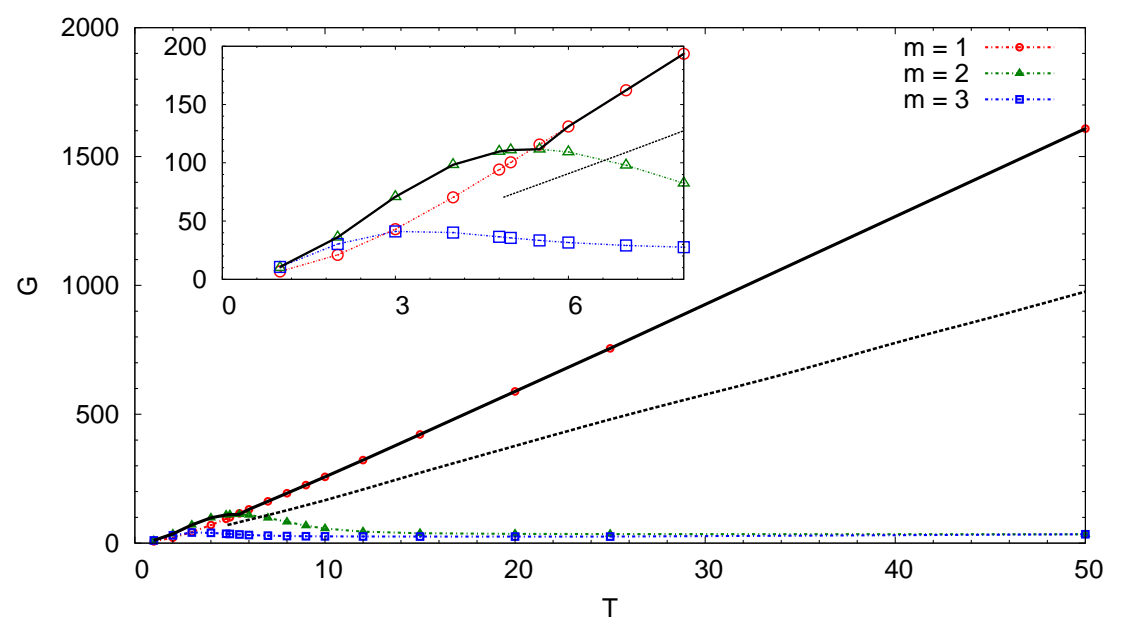

FiguRE 1. $R e=5000$ isolated vortex: variation of the largest energy gain with the horizon time for $m=1,2$ and 3. The linear optimal gain curve is the envelope of the gain curves for different $m$ and is shown by a thick solid line. Inset is a magnified view of the part of the gain curve where the linear optimal perturbation switches from $m>1$ to $m=1$. The variation of the $2 D$ linear optimal gain for $R e=1000$ and $m=1$ reported by Antkowiak \& Brancher (2004) is shown by a dashed line.

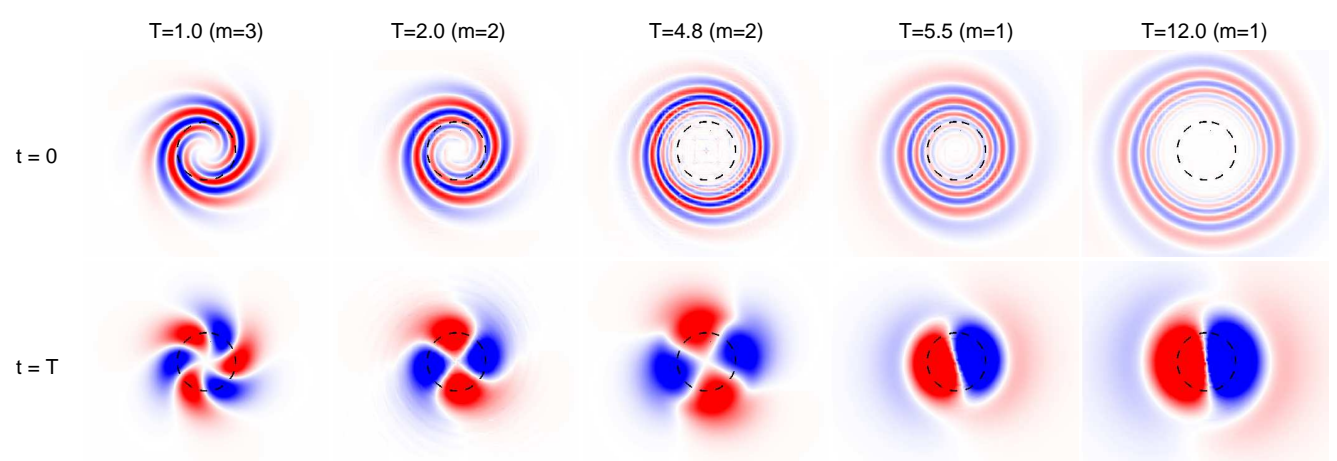

FiguRE 2. $R e=5000$ isolated vortex: vorticity field of the linear optimal perturbation for varying horizon time (top row). The corresponding vorticity field at the horizon time $t=T$ is shown in the bottom row.

where $(r, \theta)$ represents a spatial location with respect to the center of the cylindrical coordinate system, $m$ is the azimuthal wavenumber that takes non-negative integer $(\mathbb{Z})$ values and c.c. denotes the complex conjugate. In the linear framework different azimuthal components evolve independently from one another. Therefore, we carry out linear optimization for different wavenumbers $m$ separately. Linear optimal gain (LO-G) for a given horizon time $T$ is the largest gain of all azimuthal wavenumbers. Figure 1 shows the variation of largest gain with $T$ for various $m$. The horizon time has been rendered non-dimensional by the rotation period of the base flow $\left(\tau=4 \pi^{2} a^{2} / \Gamma\right)$. The LO-G curve, shown as a thick solid line in figure 1 , is the envelope of the different $m$ gain curves. It is observed that LO-G increases with $T$ up to the largest horizon time that has been studied. For $T<5.5$, the optimal perturbation has azimuthal wavenumber $m>1$. For $T \geqslant 5.5$, LO-P corresponds to $m=1$. A kink is observed in LO-G curve at $T=5.5$ that marks the shift of LO-P from $m=2$ to 1 (see the inset of figure 1 ). For 


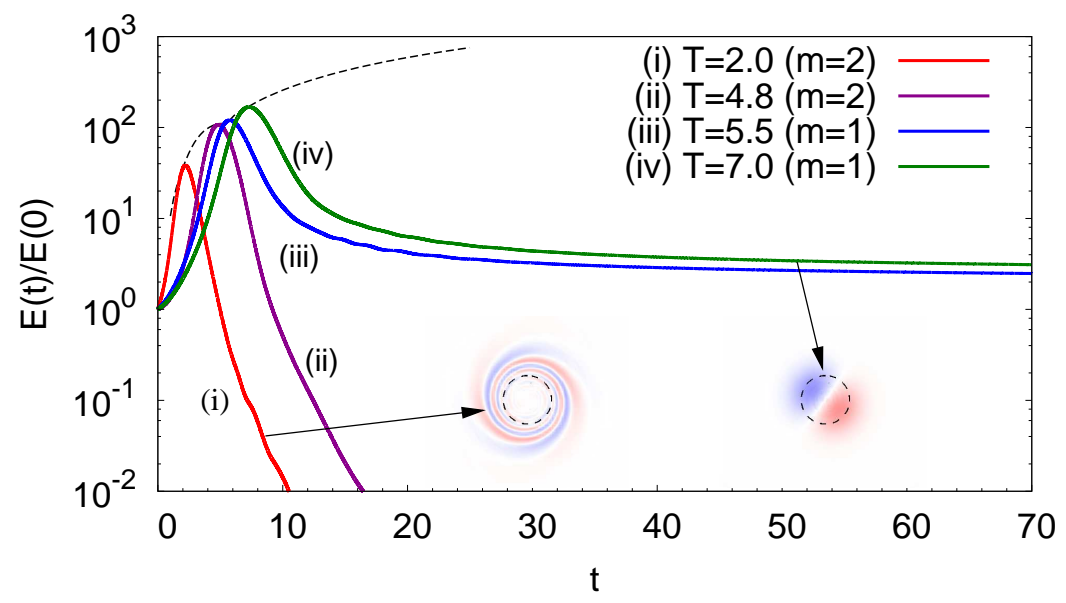

FiguRE 3. $R e=5000$ isolated vortex: evolution of the energy gain with time for computations initiated with the linear optimal perturbation corresponding to different horizon times. The azimuthal wavenumber of linear optimal perturbation is written inside parentheses in the legend. The evolution of perturbation is governed by linearized version of equation system (2.4)-(2.5). The variation of the linear optimal gain with $T$ is shown using a dashed line. The long-term perturbation vorticity field for the computations initiated with the $T=2.0$ and 7.0 linear optimal perturbations are shown in inset. The time instants from which the two fields are taken are marked by arrows.

$T \geqslant 5.5$ the optimal gain increases linearly with $T$. This is consistent with $R e=1000$ result of Antkowiak \& Brancher (2004). The gain curve reported by them is shown by a dashed line in figure 1. The slope of LO-G curve for $R e=5000$ is higher than $R e=1000$. Accordingly, as $T$ increases the difference between LO-G for the two Reynolds numbers grows.

The vorticity field associated with LO-P for different horizon time is shown in the top row of figure 2. In the bottom row, the corresponding evolved vorticity field at $t=T$ is shown. LO-P consists of vorticity filaments in spiral arrangement. Energy growth for such flow structures is attributed to the Orr mechanism (Orr 1907). The Orr mechanism is typical of plane shear flows. Its relevance in rotational flows has been demonstrated by Antkowiak \& Brancher (2004) and Pradeep \& Hussain (2006). As T increases, the vortex filaments move away from the vortex center. During the flow evolution, the vorticity filaments uncoil. For large $T$, a dipolar perturbation appears in and around the core region of the vortex. This can be observed in last column of figure 2 . The effect of the dipolar structure is to shift the vortex core from its unperturbed location. For lower horizon times $(T<5.5)$, the optimal perturbation leads to a quadrupolar $(m=2)$ or a six-polar $(m=3)$ structure which does not induce any shift in the position of the vortex core.

\subsubsection{Long-term linear evolution of the linear optimal perturbation}

Direct time integration of the linearized version of equations (2.4)-(2.5) is carried out using LO-P for various $T$ as the initial condition. The time evolution of the energy gain is shown in figure 3 . In each case, the gain reaches a peak value and then decreases rapidly. The peak gain corresponding to each $T$ is nearly the same as LO-G for the same $T$. For $T<5.5$, the gain continues to decrease rapidly even at large times. The long-term perturbation field consists of vorticity filaments similar to those of the initial perturbation but in an opposite spiral arrangement (inset of figure 3 ; c.f. figure 2 ). For $T \geqslant 5.5$ the 


\begin{tabular}{llll}
\hline$R$ & $\begin{array}{l}T=4.8 \\
\text { linear }\end{array}$ & $\begin{array}{l}T=7.0 \\
\text { linear }\end{array}$ & $\begin{array}{l}T=4.8 \\
\text { nonlinear }\end{array}$ \\
\hline $15 a$ & 109.4 & 163.6 & 140.8 \\
$26 a$ & 109.5 & 163.9 & 141.9 \\
$37 a$ & 109.5 & 164.6 & 141.9
\end{tabular}

TABLE 1. Effect of the size of the computational domain on the value of optimal gain for $T=4.8$ and 7.0. The nonlinear gains are for $E(0)=0.01 . R$ is the radius of the computational domain.
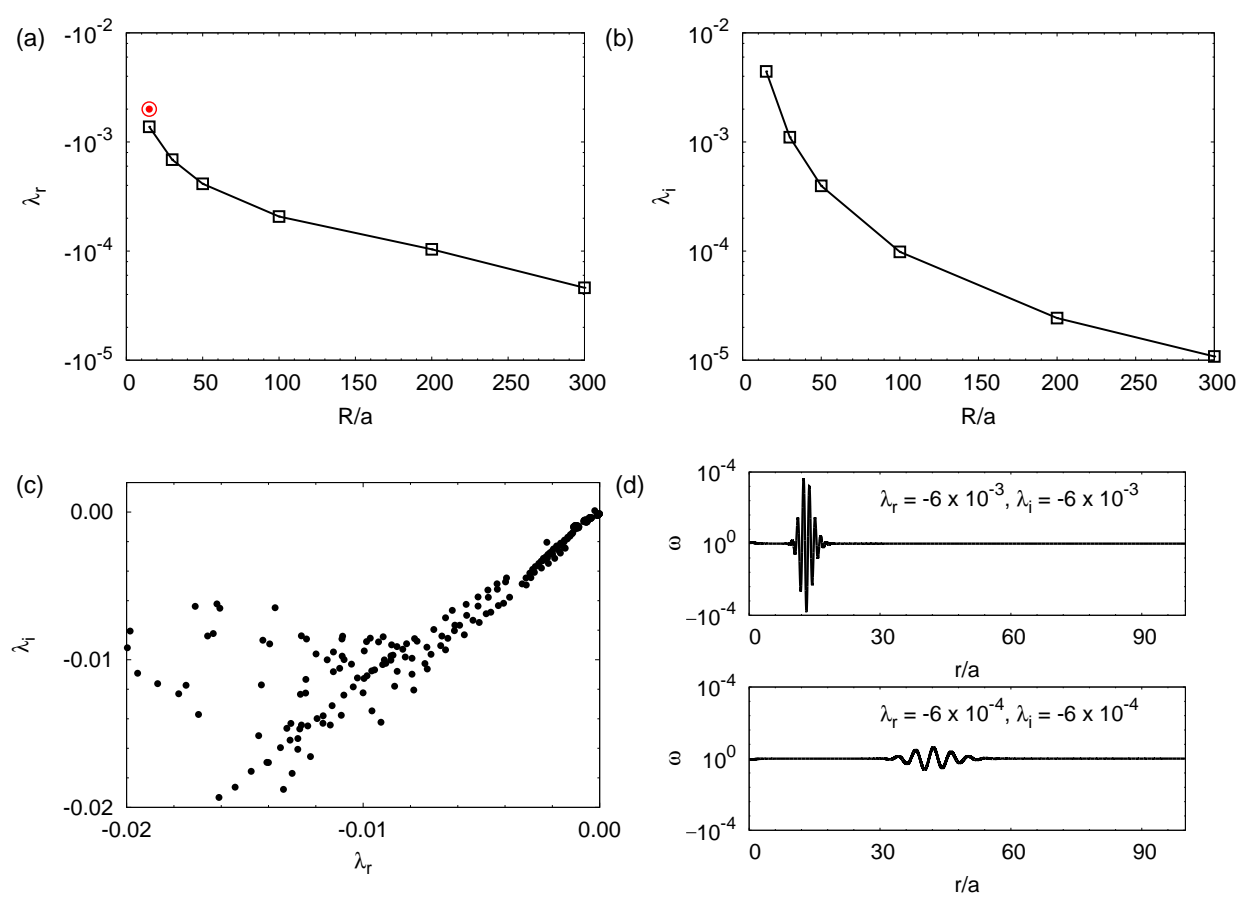

Figure 4. $R e=5000$ Linear Stability Analysis of an isolated Lamb-Oseen vortex: variation of the non-dimensional (a) growth rate and, (b) frequency of the displacement mode with the size of the computational domain. The results have been rendered non-dimensional with respect to the rotation time of the vortex $\tau$. The growth rate of the perturbation calculated using the energy decay rate at large time for $T=7.0$ in figure 3 is shown by a bull's eye symbol. (c) Part of the continuous spectrum for $m=1$. (d) Vorticity distributions for eigenmodes from the continuous spectrum corresponding to two eigenvalues. $r$ denotes radial distance from the origin of the cylindrical co-ordinate system.

rapid decay of perturbation energy seems to arrest after some time. Subsequently the energy decays very slowly and the perturbation has a dipolar structure (see inset of figure $3)$.

LO-P for a given horizon time can be written as a linear combination of the eigenmodes of the linearized Navier-Stokes operator (Pradeep \& Hussain 2006). Due to the nonorthogonality of the eigenmodes, transient growth of perturbation energy is possible in vortex systems (Antkowiak \& Brancher 2004; Pradeep \& Hussain 2006). However, longterm the most unstable or least stable mode is expected to dominate the flow evolution. 
Fabre, Sipp \& Jacquin (2006) (hereafter referred to as FSJ 2006) carried out Linear Stability Analysis (LSA) of Lamb-Oseen vortex and found that all the eigenmodes, other than the $2 D$-displacement mode $(m=1)$, have negative growth rate. The $2 D$ displacement mode is neutrally stable and its effect on the base flow is to shift the location of the vortex center. It is observed that the long-term perturbation field for $T \geqslant 5.5$ in figure 3 is very similar to the $2 D$-displacement mode. This implies that the $2 D$-displacement mode contributes to the LO-P for $T \geqslant 5.5$. As the $2 D$-displacement mode has zero growth rate, the perturbation energy at large time is expected not to vary with time. We, however, observe that the perturbation energy decays slowly at large time. The reason for this is the finiteness of the computational domain used for the present work. To elucidate this, we present the variation of the growth rate $\left(\lambda_{r}\right)$ and the frequency $\left(\lambda_{i}\right)$ of the $2 D$-displacement mode obtained via LSA (section 2.3) with the radius of the computational domain $(R)$ in figures 4 (a) and (b), respectively. It can be observed that both $\lambda_{r}$ and $\lambda_{i}$ are close but not equal to zero. Both the quantities seem to approach zero as $R \rightarrow \infty$. Due to the negative growth rate of the $2 D$-displacement mode in the finite domain, the perturbation decays long-term for $T \geqslant 5$.5. This is confirmed by noting that the decay rate derived from the energy evolution curve for $T \geqslant 5.5$ at large time (shown by bull's eye symbol in figure $4 \mathrm{a}$ ) is close to the growth rate of the $2 D$ displacement mode obtained via LSA. The difference between the two values is because of the presence of other modes, albeit with lower magnitude than the $2 D$-displacement mode. If the linearized simulations are carried on further the difference is expected to become smaller.

A question that arises in view of the sensitivity of LSA results to the extent of the computational domain is: does the size of the domain affect the value of the LO-G? Our computations show that beyond a certain radial extent, LO-G is unaffected. Table 1 lists the values of LO-G for $T=4.8$ and 7.0 for different values of domain radius $R$. The suitable size of the computational domain for optimal analysis depends on the horizon time $T$. This is because the vortex filaments of the LO-P move away from the vortex center as $T$ increases. In the present case $R=15 a$ appears to be adequate to carry out linear optimization at least up to $T=7$.

Figure 1 shows that LO-G increases monotonically with $T$. Therefore a Lamb-Oseen vortex can support transient growth up to very large horizon times, despite being linearly stable. The reason is understood to be the existence of a continuous spectrum for the Lamb-Oseen vortex. Mao \& Sherwin $(2011 ; 2012)$ found that in addition to the discrete spectrum, two continuous spectra exist for the Batchelor vortex. The tangential and radial velocity distribution of a Batchelor vortex is similar to a Lamb-Oseen vortex. However, unlike Lamb-Oseen vortex which is a $2 D$ model, Batchelor vortex is characterized by Gaussian distribution of axial velocity. As for the Batchelor vortex, continuous spectrum exists for Lamb-Oseen vortex as well. Figure 4(c) shows a part of the continuous spectrum of Lamb-Oseen vortex for $m=1$. The computations have been carried out in a domain of radial length $R=100 a$. It is observed that the modes from the continuous spectrum can have a very low decay rate. The lower the decay rate, the further the mode is located from the vortex core (figure $4 \mathrm{~d}$ ). The discrete modes are generally located near the vortex center (FSJ 2006). We recall that with increasing T, LO-P moves away from the vortex center. Therefore, LO-P at large $T$ is constituted of modes from the continuous spectrum that have a low decay rate. Consequently, the continuous spectrum and the $2 D$-displacement mode combine to exhibit transient growth of perturbation energy up to very large values of horizon time.

We end the part on linear analysis by a short note on the applicability of finite domain simulations. In practical scenarios, an isolated vortex does not exist. In most situations a 
(a)

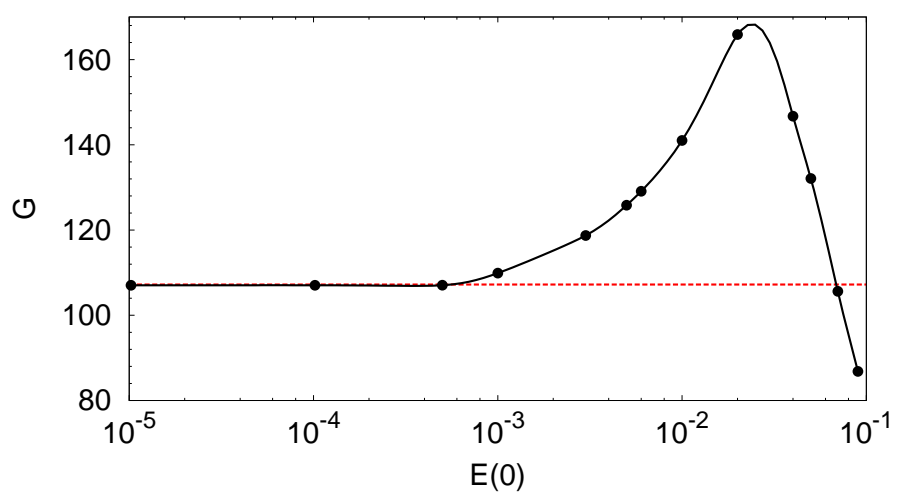

(b)

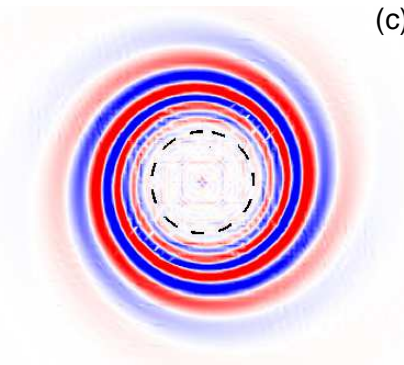

(c)

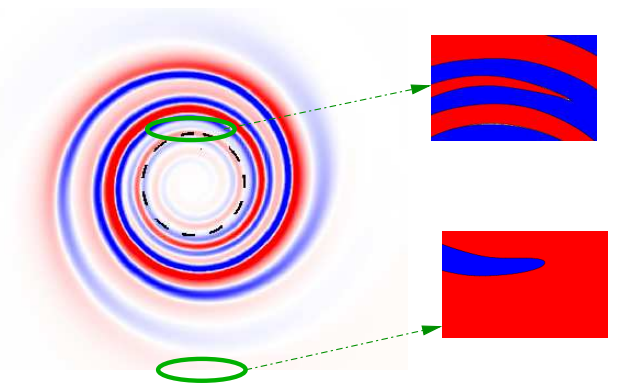

FiguRE 5. $R e=5000$ isolated vortex: (a) variation of the nonlinear optimal gain for $T=4.8$ with initial energy of the perturbation The azimuthal velocity field associated with nonlinear optimal perturbation (at $t=0$ ) for $E(0)=10^{-4}$ and $E(0)=3 \times 10^{-3}$ is shown in (b) and (c), respectively. A magnified view of the two spiral dislocations in the nonlinear optimal perturbation is provided. A synthetic two-color scheme has been used in the magnified view to display the dislocations clearly. In (a) the value of the linear optimal gain is indicated by a dashed line.

vortex belongs to a part of multi-vortex system, for example, trailing vortices in the wake of an aircraft. The effect of surrounding vortices, in such situations can be emulated by using appropriate boundary conditions, albeit in a finite domain. We would like to point out that in practice it is impossible to carry out infinite domain computations.

\subsection{Nonlinear analysis}

Recent works on nonlinear optimization of flows (like boundary layer flow, plane Couette flow) have shown that for the same horizon time nonlinear optimal perturbation (NLO-P) can result in higher gain than LO-P (Cherubini et al. 2011, 2013; Kerswell 2018). We extend nonlinear optimization to Lamb-Oseen vortex. Non-linear optimization is carried out by retaining the nonlinear terms in the direct-adjoint iterative process (section 2.2). The adjoint equations require the input of the direct perturbation field. Hence, the time history of the direct flow is saved at each iteration of the optimization process. This increases the computational cost significantly when compared with linear optimization. Unlike for LO-G, nonlinear optimal gain (NLO-G) depends on the initial energy of the perturbation $(E(0))$. Therefore the nonlinear framework presents a richer parameter space than the linear framework. For each horizon time, computations are carried out for varying values of $E(0)$. The general variation of NLO-G with $E(0)$ is found to be similar for different horizon times $T$. We have selected $T=4.8$ to present a detailed analysis. Unless stated otherwise, the results for nonlinear optimization of the isolated vortex are for $T=4.8$. 


\subsubsection{Optimal gain}

Figure 5(a) provides the variation of NLO-G with $E(0)$ for $T=4.8$. The corresponding $\mathrm{LO}-\mathrm{G}$ is shown using a dashed line in the same figure. For low values of the initial energy $\left(E(0) \leqslant 5 \times 10^{-4}\right)$, NLO-G is nearly the same as LO-G. Beyond $E(0)=5 \times 10^{-4}$, NLO-G departs from the linear value. At first, NLO-G increases with $E(0)$ and reaches a peak value for $E(0)=0.03$. The NLO-G value for $E(0)=0.03$ is $\sim 50 \%$ higher than LO-G. With further increase in $E(0)$, NLO-G decreases monotonically. $G$ becomes lower than $\mathrm{LO}-\mathrm{G}$ for $E(0) \geqslant 0.07$. Therefore, there exists a range of $E(0)$ for which NLO-P yields higher gain than the LO-P.

Table 1 demonstrates the effect of the size of the computational domain on the value of NLO-G. Similarly to LO-G, the NLO-G for $T \leqslant 7.0$ is unaffected by expansion of the domain beyond $15 a$.

Cherubini et al. (2013) investigated NLO-P for the plane Couette flow (PCF) and reported a similar variation of NLO-G with $E(0)$ as in figure $5(\mathrm{a})$. Unlike in the present work they considered $3 D$ perturbations in their analysis. We note that the base state for both flows is $2 D$ and linearly stable. In both flows, there exists a threshold value of $E(0)$ below which NLO-G remains nearly the same as LO-G; above the threshold value, NLO-G shows significant departure from LO-G. However, unlike the PCF where NLO-G remains higher than LO-G for $E(0)$ greater than the threshold, NLO-G for a $2 D$ LO vortex becomes lower than the LO-G for large values of $E(0)$. The explanation may be that in the PCF, for large values of $E(0)$, the flow field at the horizon time becomes turbulent. With the restriction of $2 D$ perturbation for Lamb-Oseen vortex, there is no possibility of flow transition even with large $E(0)$, and any initial perturbation must eventually decay as suggested by the result of linear stability analysis.

\subsubsection{Optimal perturbation}

Figures 5(b) and (c) give the vorticity fields associated with NLO-P for $E(0)=10^{-4}$ and $3 \times 10^{-3}$, respectively. We recall that the LO-P for $T=4.8$ has azimuthal wavenumber of $m=2$ (figure 1). The shape of NLO-P for $E(0)=10^{-4}$ is very similar to that of the $m=2$ linear optimal. Thus, for low $E(0)$ both NLO-G as well as the shape of NLO$\mathrm{P}$ is similar to that obtained via linear analysis. This is so because the contribution of the nonlinear terms to the evolution of the perturbation becomes smaller as $E(0)$ tends to zero. Beyond the threshold energy $\left(E(0)>5 \times 10^{-4}\right)$ two major differences are observed with respect to the LO-P. First, the vorticity filaments far from the vortex core appear less diffused. Second, two spiral dislocations are observed. A spiral dislocation is identified by the merger of two vorticity filaments of the same sign. In figure $5(\mathrm{c})$, the locations of the two spiral dislocations is shown by solid ellipses. The dislocations are situated at distances of $\sim 1.1 a$ and $\sim 3.6 a$ from the vortex center. A magnified view of the two dislocations using a synthetic two-color scale is provided in figure $5(\mathrm{c})$. In the first dislocation $(r \sim 1.1 a)$ two negative vorticity filaments, that are separated by a positive vorticity filament, merge. In the second dislocation $(r \sim 3.6 a)$ two positive vorticity filaments merge. The general shape of NLO-P for $E(0)>5 \times 10^{-4}$ is similar.

Figure 6 shows the relative contribution of different azimuthal wavenumbers to the $E(0)=3 \times 10^{-3}$ NLO-P as a function of distance from the vortex center. The contribution of $|m| \geqslant 4$ is relatively small and hence are not included in the figure. The data has been generated by using Fourier decomposition of the azimuthal component of the perturbation velocity for several radial locations. Unlike LO-P, NLO-P has contribution from different azimuthal wavenumbers. At any given radial location, the azimuthal mean of the velocity is non-zero as indicated by the presence of $m=0$ component. Among the non-zero wavenumbers, the most significant contribution to NLO-P is from $m=1$ 


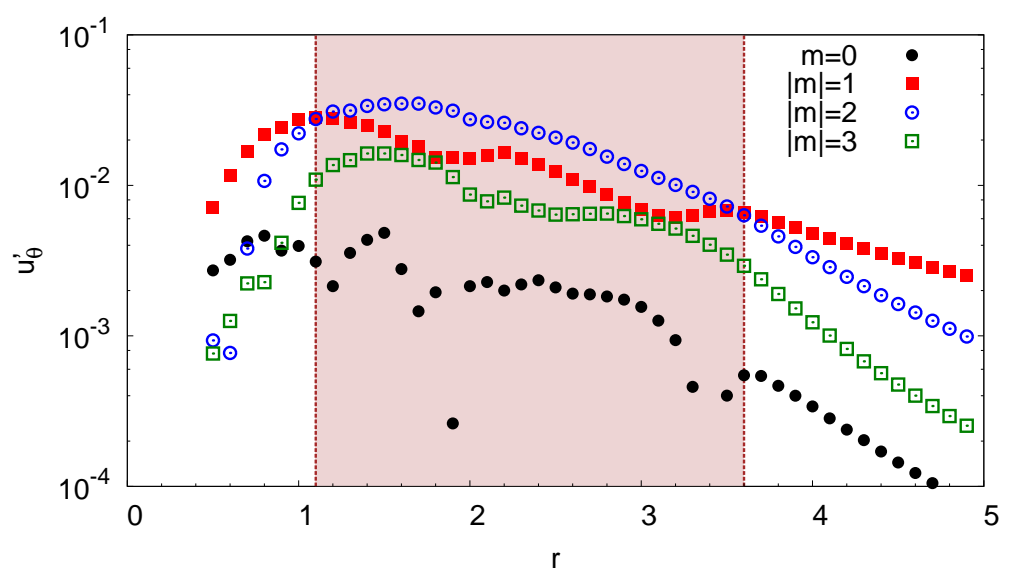

FiguRE 6. $R e=5000$ isolated vortex: Fourier decomposition of the azimuthal component of the $T=4.8, E(0)=3 \times 10^{-3}$ nonlinear optimal perturbation $(t=0)$ velocity field at various radial locations. $m$ is the azimuthal wavenumber. The shaded part corresponds to the radial segment between the two spiral dislocations in the nonlinear optimal perturbation shown in figure 5(c).

and 2. The shaded region in figure 6 corresponds to the radial location between the two spiral dislocations in NLO-P (figure 5(c)). It is observed that in the shaded part $m=2$ is the most dominant component, whereas outside the shaded part the most dominant wavenumber is $m=1$. It appears that spiral dislocation is the consequence of the switch in the dominant wavenumber within the NLO-P.

Recently, Bisanti (2013) investigated NLO-P for a $2 D$ Lamb-Oseen vortex. The work was restricted to $m=2$ perturbations. For $T=4.8$ and $E(0)=0.01$ Bisanti (2013) found the value of NLO-G to be lower than the LO-G. Our computations show that for the same parameters NLO-G is higher than the LO-G (figure 5a). Figure 7(a) shows the variation of energy gain with iteration number during the optimization process for $T=4.8$ and $E(0)=0.01$. It is observed that close to the value of the optimal gain reported by Bisanti (2013) the variation of gain seems to plateau (point A); the increment in the value of optimal gain with each iteration $(J)$ is $\mathcal{O} \sim 10^{-3}$ and it decreases near point A (see figure $7 \mathrm{~b}$ ). We refer to point $\mathrm{A}$ as a sub-optimal state. Figure $7(\mathrm{c})$ shows the vorticity field corresponding to the optimal perturbation and its evolved state at $t=T$ as reported by Bisanti (2013). Figure 7(d) gives the same field for the sub-optimal state obtained in the present work. The fields are very similar, therefore the sub-optimal state corresponds to the $m=2$ optimal state reported by Bisanti (2013). If the iterations are continued further, $J$ starts to increase again. The value of gain increases with $N_{i t e r}$ and a converged state (point B) is reached with higher energy gain. Beyond point $B$, energy gain does not change significantly with $N_{i t e r}$ up to $N_{i t e r} \sim 500$, which is when we terminate the nonlinear optimization process. The value of $J$ in the converged state is $\mathcal{O} \sim 10^{-5}$. As shown in figure 6, NLO-P for $T=4.8$ and $E(0)=0.01$ has, in addition to $m=2$, significant contributions from other azimuthal wavenumbers. The difference between the optimal gain reported by Bisanti (2013) and NLO-G of the present work is therefore attributed to nonlinear interactions between different azimuthal components. We would like to mention here that in nonlinear optimization with direct-adjoint technique, it is not possible to completely ascertain if point B is the global optimum. We carried out $T=4.8, E(0)=0.01$ nonlinear optimization with different initial guesses, for example, random perturbation, linear/nonlinear optimal perturbation for different $T$. In each case 

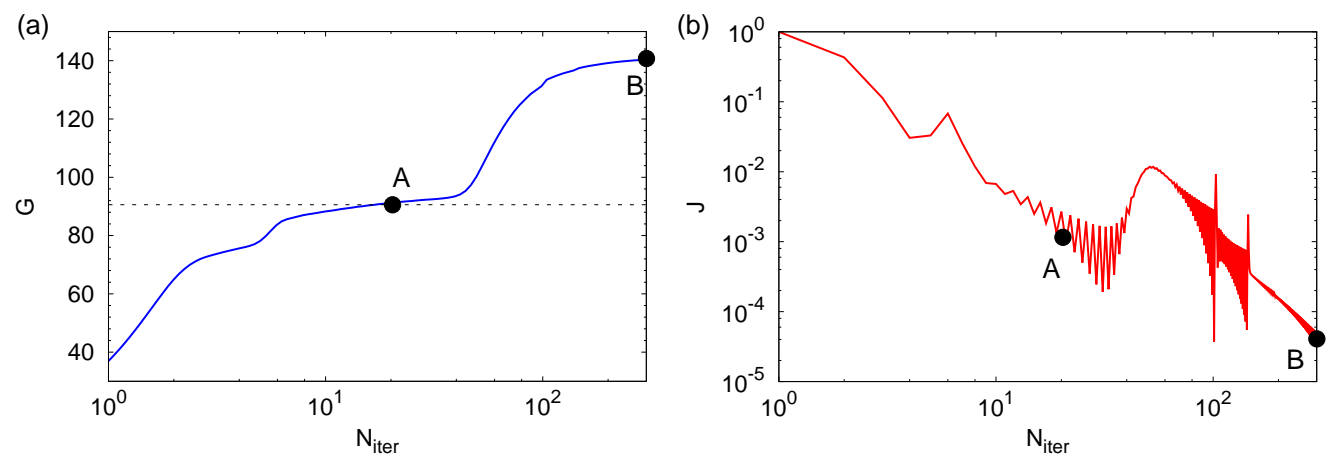

(c)

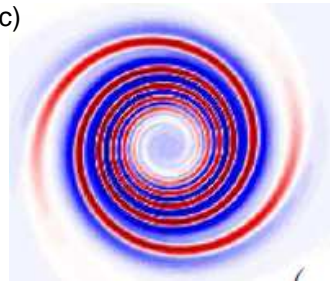

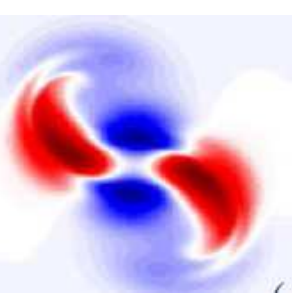

(d)

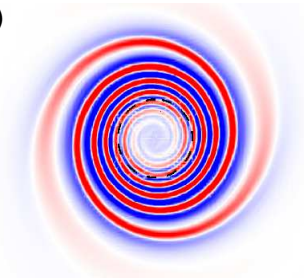

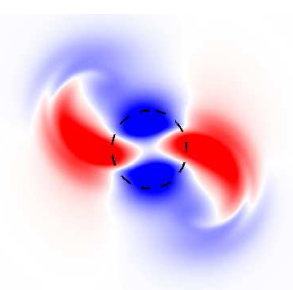

FiguRE 7. $R e=5000$ isolated vortex: variation of (a) the nonlinear optimal gain and (b) the increment in gain with iteration number during the optimization process for $T=4.8$ and $E(0)=0.01$. The sub-optimal and optimal states are labeled $A$ and $B$ respectively. The optimal gain reported by Bisanti (2013) is indicated by a dashed line in (a). (c) Vorticity field for the $m=2$ optimal perturbation and its evolved state at $t=T$ as reported by Bisanti (2013). (d) Vorticity field for sub-optimal perturbation and its evolved state at $t=T$ as obtained in present work.
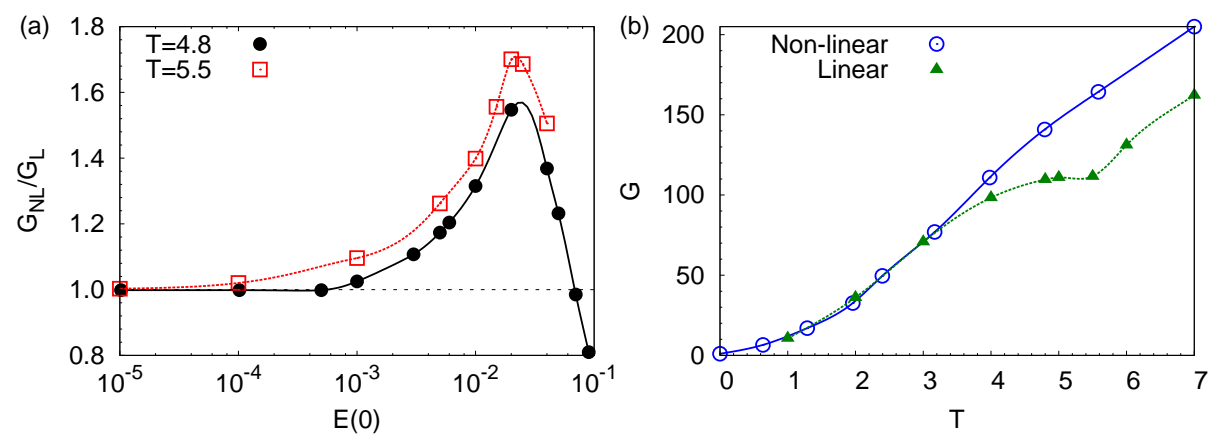

FIGURE 8. $R e=5000$ isolated vortex: variation of (a) the ratio between the nonlinear optimal gain and the LO-G with initial energy of the perturbation for $T=4.8$ and 5.5 and (b) linear and $E(0)=0.01$ nonlinear optimal gain with horizon time.

the converged solution corresponds to point B. It is, therefore, likely that point B is the global optimum, and that there is no jump in energy gain beyond point B in figure $7(\mathrm{a})$.

\subsubsection{Effect of the horizon time}

Figure 8(a) displays the effect of $T$ on NLO-G. For a given initial energy of the perturbation, NLO-G increases with $T$. The peak optimal gain for different $T$ occurs for nearly the same value of $E(0)$. It is observed that the threshold energy below which the NLO-G and the LO-G are nearly the same decreases with increasing $T$. The threshold energies for $T=4.8$ and 5.5 are $10^{-3}$ and $10^{-4}$ respectively. Figure $8(\mathrm{~b})$ shows the 

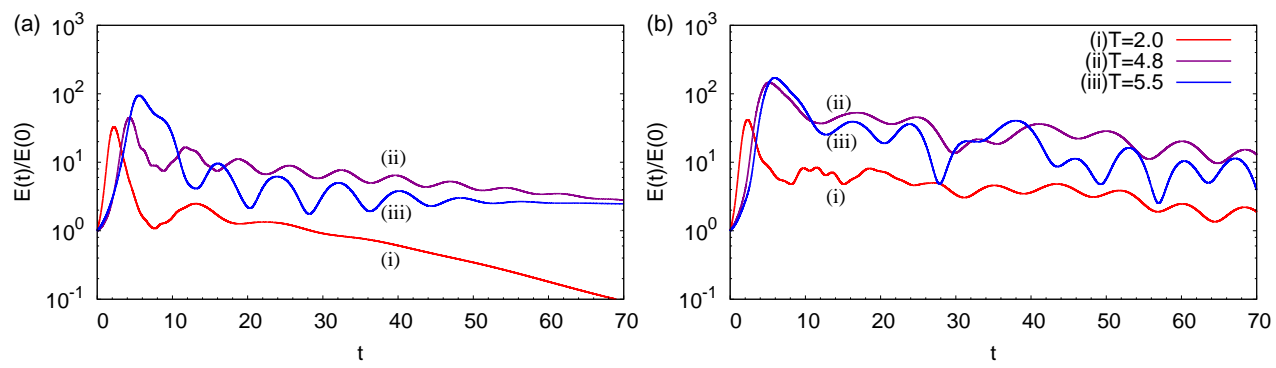

FiguRE 9. $R e=5000$ isolated vortex: evolution of energy gain with time for computations initiated with (a) linear optimal perturbation and (b) $E(0)=0.01$ nonlinear optimal perturbation, for different horizon times. The evolution of perturbation is governed by equation system (2.4)-(2.5).

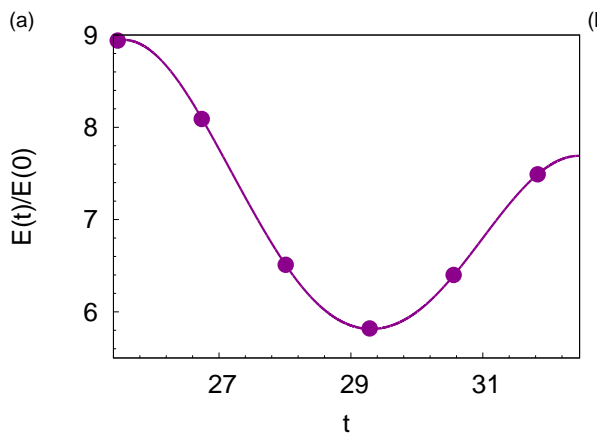

(b)

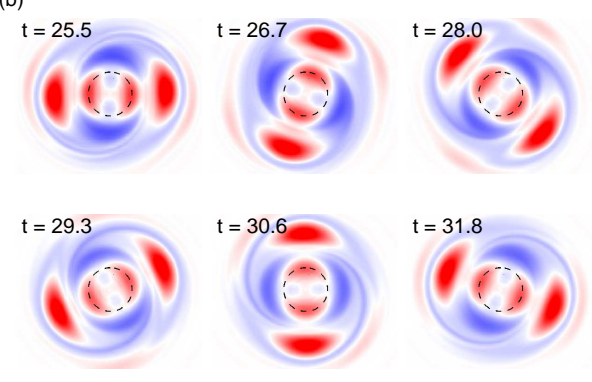

FiguRE 10. $R e=5000$ isolated vortex: (a) variation of the energy gain during one rotation period of the quasi-steady non-axisymmetric state for computations initiated with the $T=4.8$ LO-P. (b) The perturbation vorticity field at various time instants marked by solid circles in the gain curve.

variation of NLO-G for $E(0)=10^{-2}$ with horizon time. For $T \leqslant 3.0$, the linear and nonlinear optimal gain is nearly the same. This implies that the threshold energy for $T \leqslant 3.0$ is higher than $10^{-2}$. For $T>3.0$, the threshold energy is lower than $10^{-2}$ and the linear and nonlinear optimal gains are different. The difference between LO-G and NLO-G increases with $T$ up to $T=7.0$. The threshold energy decreases with increasing $T$.

\subsubsection{Long-term nonlinear evolution of linear and nonlinear optimal perturbation: appearance of a quasi-steady non-axisymmetric vortex}

Rossi, Lingevitch \& Bernoff (1997) carried out numerical simulations to investigate the evolution of non-axisymmetric perturbations to $2 D$ Lamb-Oseen vortex. They observed that for weak non-axisymmetric perturbation, the vortex relaxes towards an axisymmetric state. If, however, the perturbation is strong enough, a quasi-steady non-axisymmetric vortex state can be reached. Quasi-steady vortex, as defined by Rossi, Lingevitch \& Bernoff (1997), is one that maintains approximately the same relative distribution of vorticity over several rotation periods of the vortex and diffuses on a slow time scale. The results presented in the previous sections show that linear and nonlinear optimal perturbation can result in a large energy gain at horizon time. Furthermore, the evolved perturbation field at horizon time is non-axisymmetric. Therefore it is plausible that as the perturbation relaxes from its high energy state, the vortex might exhibit quasi-steady non-axisymmetric behavior. To explore this, we carry out direct time integration of the 
(a)

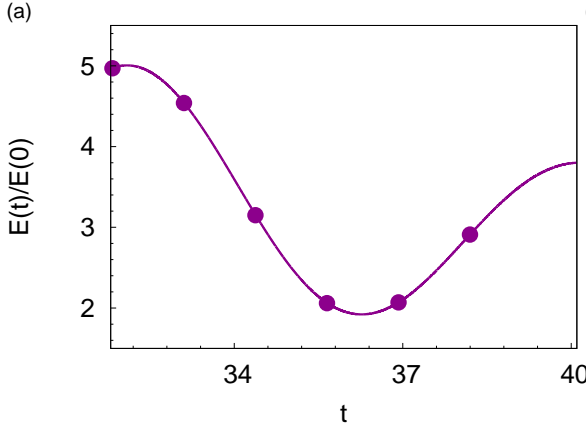

(b)

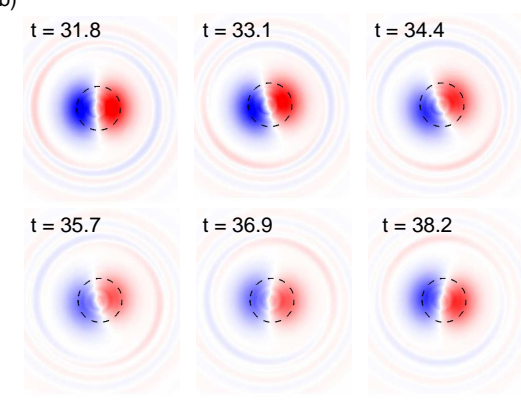

FiguRE 11. $R e=5000$ isolated vortex: (a) variation of the energy gain during one rotation period of the quasi-steady non-axisymmetric state for computations initiated with the $T=5.5$ LO-P. (b) The perturbation vorticity field at various time instants marked by solid circles in the gain curve.
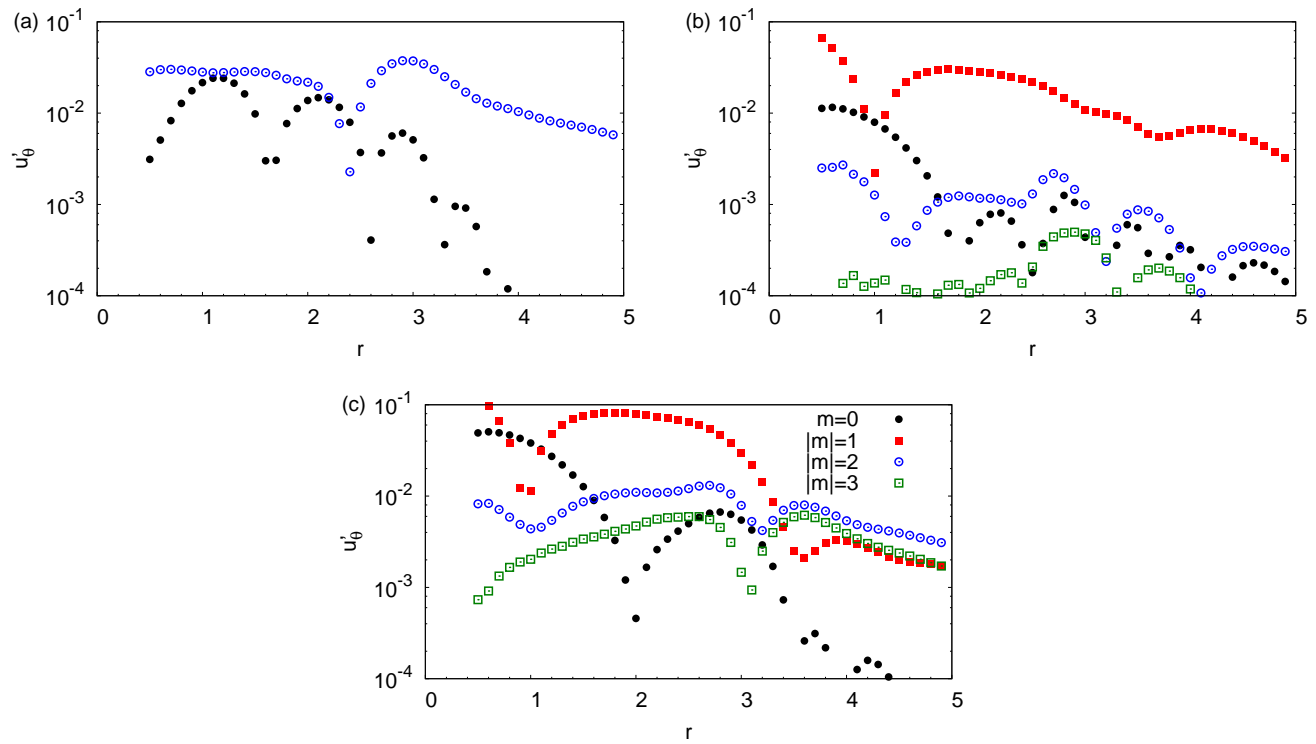

Figure 12. $R e=5000$ isolated vortex: Fourier decomposition of the azimuthal component of perturbation velocity in the azimuthal direction at a time instant in the quasi-steady non-axisymmetric state for computations initiated with (a) the $T=4.8 \mathrm{LO}-\mathrm{P}$, (b) the $T=5.5$ LO-P, (c) the $T=4.8, E(0)=0.01$ NLO-P. The time instants are $t=25.5,31.8$ and 59.9 respectively.

equations governing the nonlinear evolution of the perturbations (equation (2.4)-(2.5)). The computations are initiated with optimal perturbations, linear and nonlinear, for various $T$.

First, we present results from computations initiated with LO-P. The initial perturbation energy for all of the computations is $E(0)=10^{-2}$. Figure 9 (a) shows the temporal evolution of the perturbation energy for $T=2.0,4.8$ and 5.5 LO-P. The perturbation energy $(E)$ has been normalized by $E(0)$. Initially, $E$ grows in time and its evolution is very similar to that obtained via linear simulation (figure 3 ). It attains a peak value near the horizon time. The peak value of $E$ obtained from the nonlinear computation is lower than that of the linear computation. The differences between the 


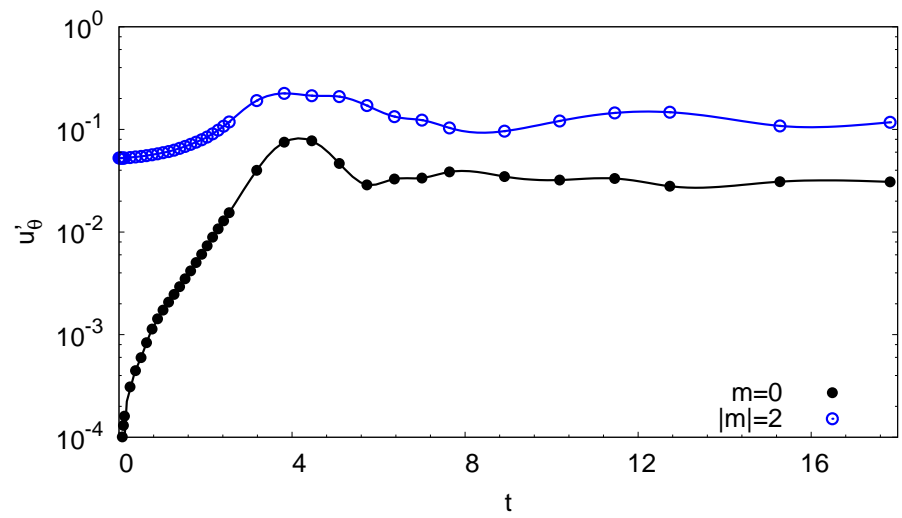

FiguRE 13. $R e=5000$ isolated vortex: variation of the contribution of different azimuthal components of perturbation velocity in the azimuthal direction with time, for nonlinear simulation initiated with $T=4.8 \mathrm{LO}-\mathrm{P}$ and $E(0)=0.01$. Key is the same as in figure 12 .
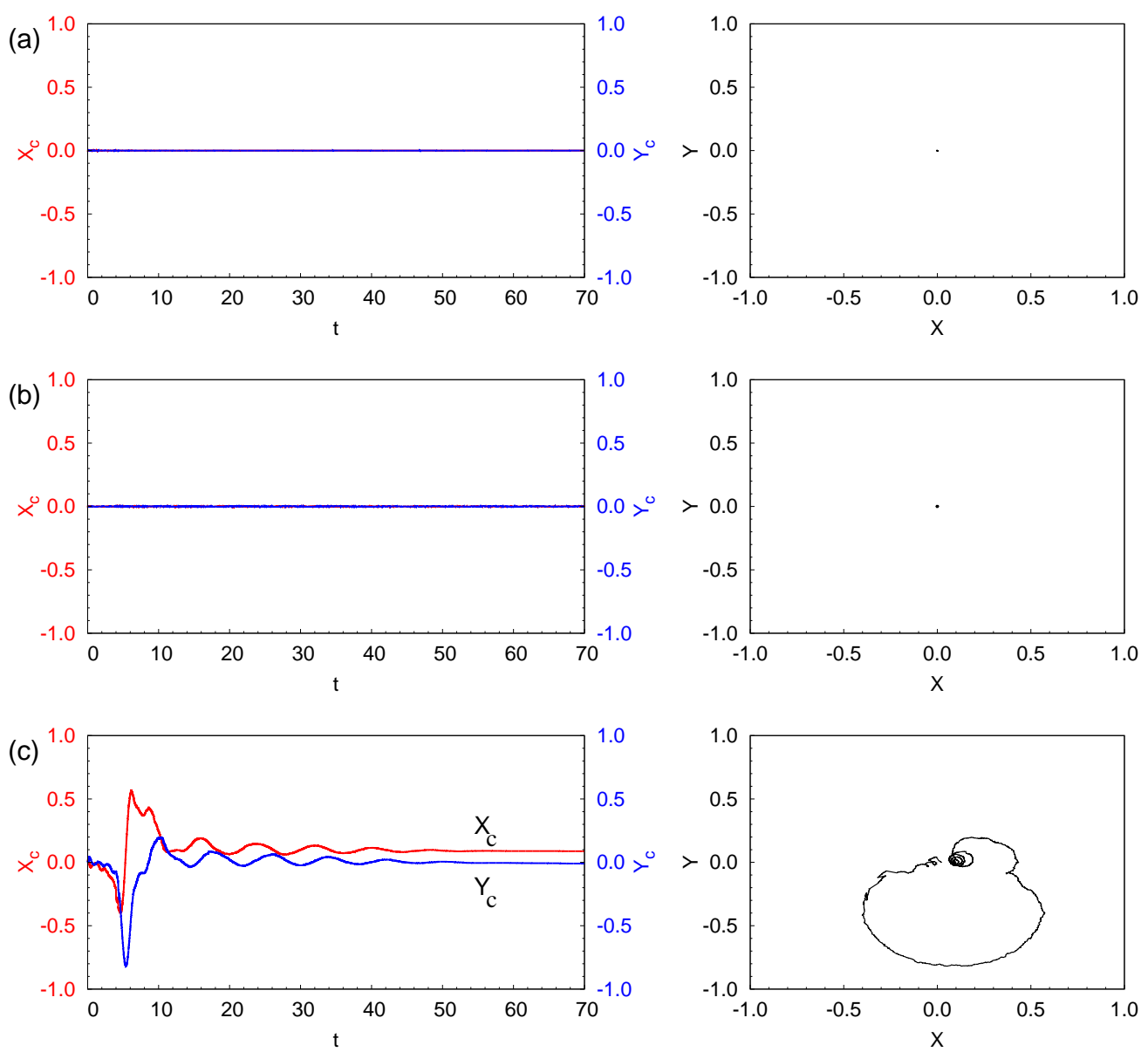

Figure 14. $R e=5000$ isolated vortex: (left column) displacement of the vortex center with time for computations initiated with the LO-P for (a) $T=2.0$, (b) $T=4.8$ and (c) $T=5.5$; (right column) corresponding trajectory of the vortex center in the $X-Y$ plane. 
peak linear and nonlinear perturbation energies for $T=2.0,4.8$ and 5.5 are $14 \%, 58 \%$ and $21 \%$ respectively. After reaching the peak, $E$ decreases for some time and then exhibits fluctuations. For $T=2$, the fluctuations decay rapidly and thereafter $E$ decreases monotonically with time. However for $T=4.8$ and 5.5 the energy fluctuations persist for a long time (more than 40 rotation periods of the vortex) and are quasi-periodic. Two time scales can be identified for $T=4.8$ and 5.5. The slow time scale corresponds to the decrease in the mean value of perturbation energy per cycle of energy fluctuation. The fast time scale corresponds to the time period of energy fluctuation. Figures 10(a) and 11(a) show the variation of energy on the fast time scale for $T=4.8$ and 5.5 respectively. The fast time scale is attributed to energy exchange between the base flow and perturbation. During one half of the cycle, the perturbation draws energy from the base flow, and in the other half the perturbation returns energy to the base flow. The periodic fluctuation of perturbation energy is associated with the quasi-steady non-axisymmetric vortex state (discussed in next paragraph). Since viscous effects in the quasi-steady state take place on the slow time scale (Rossi, Lingevitch \& Bernoff 1997) we attribute the decrease in mean energy per cycle of energy fluctuation to viscosity.

Figure 10(b) shows the perturbation vorticity field at different time instants over a cycle of energy fluctuation for $T=4.8$. The perturbation has non-axisymmetric structure; at least six regions of concentrated vorticity can be identified in the figure. A Fourier decomposition of the perturbation field in the azimuthal direction shows that only the $m=0$ (axisymmetric) and $m=2$ components are significant (figure 12a). We recall that in the linear framework the evolution of different azimuthal components occur independently from one another. Hence, during linearized evolution of $T=4.8$ LO$\mathrm{P}$, the perturbation flow at any time instant is $m=2$. Nonlinear effects, on the other hand, may allow transfer of energy between different azimuthal components. To monitor the transfer of energy from $m=2$ component to other components during nonlinear evolution of $T=4.8$ LO-P, we plot the time evolution of the relative contribution of different azimuthal components to the perturbation flow. The plots are shown in figure 13. It is observed that as the flow evolves with time, axisymmetric component in the flow becomes comparable to $m=2$ component beyond $t \sim 2$. The contribution of other azimuthal components remains relatively small during the flow evolution (and are not shown in figure 13). The perturbation field (figure 10(b)) rotates in time and the relative orientation of the vorticity patches remain the same during rotation. The rotation period of the perturbation is equal to the time-period of energy fluctuation. As the mean energy decreases on a slow time scale, the non-axisymmetric perturbation remains in the flow for several rotation periods. Such a perturbation state, based on the definition used by Rossi, Lingevitch \& Bernoff (1997), corresponds to a quasi-steady non-axisymmetric perturbation. Figure 11(b) shows the perturbation vorticity field during a cycle of energy fluctuation for the $T=5.5$ case: a quasi-steady non-axisymmetric state exists for this case as well. The perturbation field is dominated by $m=1$ component (figure $12 \mathrm{~b}$ ). Near the vortex center the perturbation has dipolar structure. The dipole does not rotate completely about the vortex, instead it exhibits small rotational oscillations about the vortex center. The oscillation manifests in the movement of vortex core. This can be observed in figure 14(c)which shows the time history of the location of the vortex center. The location of vortex center is the barycenter of vorticity for the total flow, that is, base flow added with perturbation. The left column of figure 14 shows the displacement of the vortex center along the $X$-and $Y$-axis, and the right column shows the trajectory of the vortex center. The $X$ - and $Y$-axis are oriented along the horizontal and vertical directions with the origin at the center of the unperturbed vortex. Unlike in the $T=5.5$ 

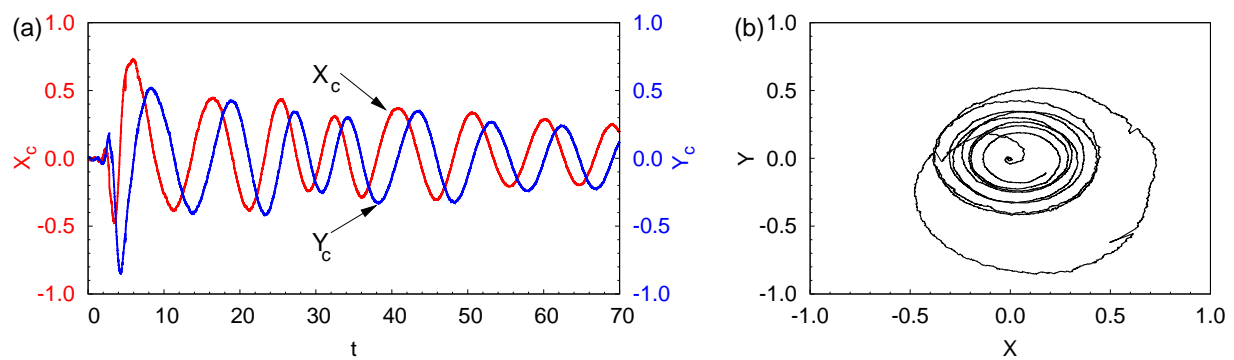

FiguRe 15. $R e=5000$ isolated vortex: (a) displacement of the vortex center with time for computations initiated with the $T=4.8, E(0)=0.01$ nonlinear optimal perturbation; (b) trajectory of the vortex center in the vortex plane.

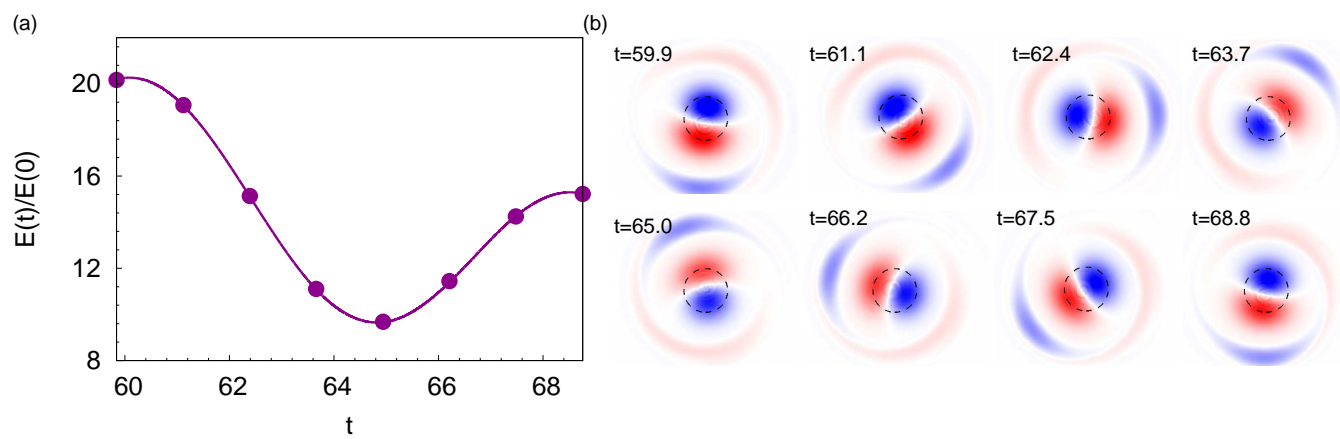

FIGURE 16. $R e=5000$ isolated vortex: (a) variation of the energy gain during one rotation period of the quasi-steady non-axisymmetric state for computations initiated with the $T=4.8$, $E(0)=0.01$ nonlinear optimal perturbation. (b) The perturbation vorticity fields at the time instants marked by solid circles in the gain curve are provided.

case, the vortex center does not move significantly for the $T=2.0$ and 4.8 cases (figures $14 \mathrm{a}, \mathrm{b})$.

Next we present results from computations initiated with the $E(0)=0.01$ NLO-P. Figure 9(b) gives the evolution of the perturbation energy for $T=2.0,4.8$ and 5.5. Similarly to the LO-P, initially the energy increases and then fluctuates with time. However, the energy fluctuation in the case of the NLO-P persists for a longer time than that of the LO-P. For example, for $T=4.8$ the energy fluctuations are observed up to $t=68$ for the LO-P, while for the $E(0)=0.01$ NLO-P fluctuations persist up to $t=160$. Figure $15(\mathrm{a})$ shows the time history of the location of vortex center along the $X-$ and $Y$ - axis with time for the $T=4.8$ NLO-P. It can be observed that the displacement of the vortex center in the two directions is more accentuated than the situation where the simulations are initiated with the LO-P. The vortex center follows a spiral trajectory about its original unperturbed location (figure 15(b)). Figure 16(b) provides the perturbation vorticity field at various time instants in a cycle of energy fluctuation. Close to the vortex core, the perturbation has a dipolar structure and is dominated by the $m=1$ component. Far from the center other non-zero wavenumbers become significant as well (figure 12c). Outside the vortex core region, two vorticity patches can be identified. The strength of the positive vorticity patch is lower than that of the negative one and of the dipole present close to the vortex center, as is evident from figure 16(b). Thus the perturbation field can be considered as a tripole consisting of a dipole and negative satellite vortex. The tripole rotates around the vortex center 
(a)

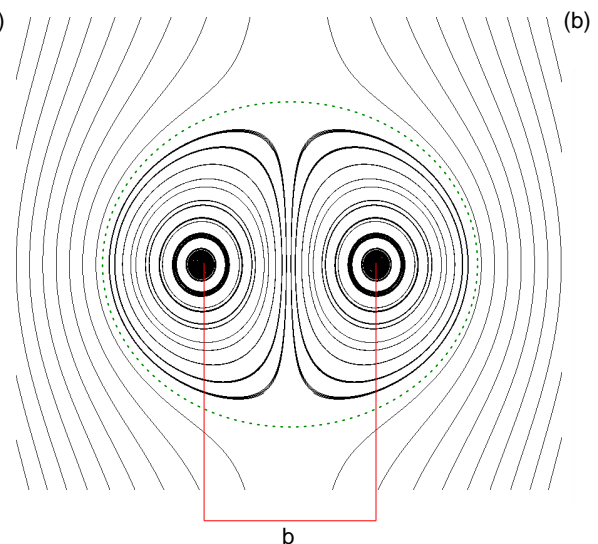

(b)

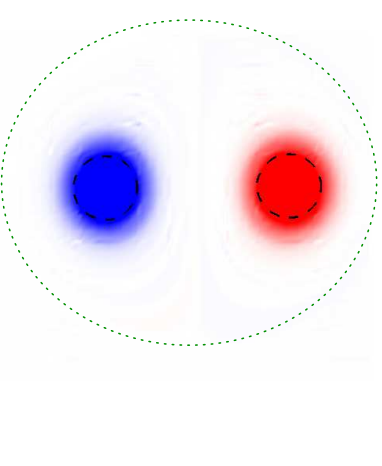

Figure 17. $R e=1000$ vortex pair: (a) streamlines and (b) vorticity field for the base flow. The Kelvin oval is indicated using dotted lines in the two figures. The vortex separation is denoted by $b$. The approximate boundary of the two vortex core regions is indicated by dashed circle in (b). The radius of the circle is equal to the vortex dispersion radius calculated using equation $(3.3)$.

and has a rotation period equal to the time period of energy fluctuation. Interestingly, the angular speed of the vorticity patch that is present outside the vortex core is close to the azimuthal velocity of the base flow at that location. This condition corresponds to the criterion of existence of critical layers in shear flows. In a critical layer, the nonlinear terms and/or the viscosity of the flow are significant and allow to deal with singularities of an otherwise inviscid description of the flow. Habermann (1972) gave a criterion to distinguish between nonlinear and linear critical layers. As per the criterion, a critical layer is nonlinear if $\epsilon^{3 / 2} R e>>1 ; \epsilon$ is a measure of the perturbation amplitude and $R e$ is the Reynolds number. If $\epsilon^{3 / 2} R e<<1$ the critical layer is of the viscous type. For $\epsilon^{3 / 2} R e \sim 1$ both nonlinear and viscous effects are significant in the critical layer. We use the Habermann criterion to identify the nature of the critical layer in figure 16 . The tripolar perturbation structure is observed after the perturbation reaches its peak energy. Therefore it is reasonable to assume that $\epsilon \sim \mathcal{O}(100)$. Consequently, $\epsilon^{3 / 2} \operatorname{Re} \sim \mathcal{O}\left(10^{5}\right)$ : the critical layer observed in figure 16 is nonlinear. We suspect that the long-term persistence of the energy fluctuation might be attributed to the role of the nonlinear critical layer. This, however, has not been investigated further as part of the present work.

\section{Vortex pair}

Our principle objective for investigating optimal perturbation of isolated vortex is its possible application in hastening the decay of aircraft trailing vortices. In general, a trailing vortex system consists of a pair of counter-rotating vortices (in some situations there can be additional vortices). It has been shown in earlier studies that a vortex can experience significant modification to its shape under the strain field of its counterrotating pair. A question then arises is, can the optimization results for isolated vortex be extended to counter-rotating vortex air? We explore the answer to this question by carrying out linear and nonlinear optimization of a vortex pair.

\subsection{Base flow}

The base flow is a pair of equal strength, counter-rotating vortices. The base flow is created by initializing the flow with a pair of counter-rotating Lamb-Oseen vortices and 
letting it evolve for some time. Since the LO-vortex pair is not a solution of the NavierStokes equations, this evolution allows the flow to adjust. During this transient period the shape of the vortices changes from axisymmetric to elliptic. The flow then evolves on a diffusive time scale. This approach has been used in earlier works on counter-rotating vortex pair (Sipp, Jacquin \& Cossu 2000; Brion 2009). Owing to its symmetry, we consider only one side of the flow for characterizing the vortex pair. Let the half-domain on the side of the counter-clockwise rotating vortex (positive vorticity) be denoted by $\Omega^{+}$. The location of the vortex center, with respect to any origin, is given by:

$$
\mathbf{r}_{\mathbf{c}}=\frac{\int_{\Omega^{+}} \mathbf{r} \omega d \Omega}{\Gamma}
$$

where $\mathbf{r}$ is the position vector with reference to the same origin. The circulation $(\Gamma)$ and vortex dispersion radius $(a)$ are given by equations (3.2) and (3.3) respectively, where the domain of integration is $\Omega^{+}$. Because of self-induction, the vortex pair translates at a speed equal to $\Gamma / 2 \pi b ; b$ is the distance between the two vortex centers. The simulations have been carried out in a frame of reference that moves with the vortex pair. Uniform velocity of magnitude $\Gamma / 2 \pi b$ is specified at the inlet boundary and a stress-free condition is imposed on the outflow and side boundaries. As for the isolated vortex, the base flow for the analysis of the vortex pair is assumed to be frozen. The ratio between the vortex dispersion radius and separation between vortex centers is $a / b \sim 0.18$. The length and velocity scales have been rendered non-dimensionalized using $b$ and $\Gamma / 2 \pi$. The Reynolds number for all vortex pair simulation is $R e=(\Gamma / 2 \pi \nu)=1000$. Figure 17 shows the streamlines and the vorticity field for the base flow. Two flow regions can be identified in the figure. In the first region, the streamlines are closed. In the second region, the flow has an open trajectory. The two flow regions are separated by a limiting streamline referred to as the Kelvin oval.

\subsection{Linear optimization}

Unlike the single vortex system, a pair of $2 D$-counter-rotating vortices is linearly unstable (Brion et al. 2014). Long-term the most unstable mode will dominate the perturbation evolution. However, before the exponential behavior sets in, transient (algebraic) growth of perturbation energy may occur due to the non-normality of the linearized NavierStokes operator. Therefore, it is possible that for some initial perturbations the energy gain at a given horizon time is higher than the gain obtained using the most unstable mode as the initial perturbation (see Appendix B). For the optimization process, as the computations are carried out in the moving reference frame, homogeneous Dirichlet boundary conditions are specified at the inlet and a stress-free condition is applied at the outflow and side boundaries for the direct equations. For the adjoint equations, a homogeneous Dirichlet boundary condition is applied on the outlet and stress-free conditions on the inlet and side walls. Figure 18(a) shows the variation of the LO-G with horizon time $(T)$ on log-lin axes. Time is rendered non-dimensional using the time taken by system of two line vortices of strength $\pm \Gamma$ to translate a distance equal to $b$ under self-induction $\left(\tau_{b}=2 \pi b^{2} / \Gamma\right)$. Also indicated by a dotted line is the energy gain corresponding to the most unstable mode. It can be observed that the difference between the two gains increases with $T$ up to $T=4.0$. For $T>4.0$, LO-G increases exponentially with $T$ according to the growth rate of the most unstable mode; the exponential growth appears as linear curve on log-lin axes. Therefore, the maximum duration for which transient processes contribute to the linear optimal gain is $4 \tau_{b}$.

Any perturbation to a vortex pair can be split into symmetric and anti-symmetric components. The velocity field for the (anti-)symmetric component is (anti-)symmetric 


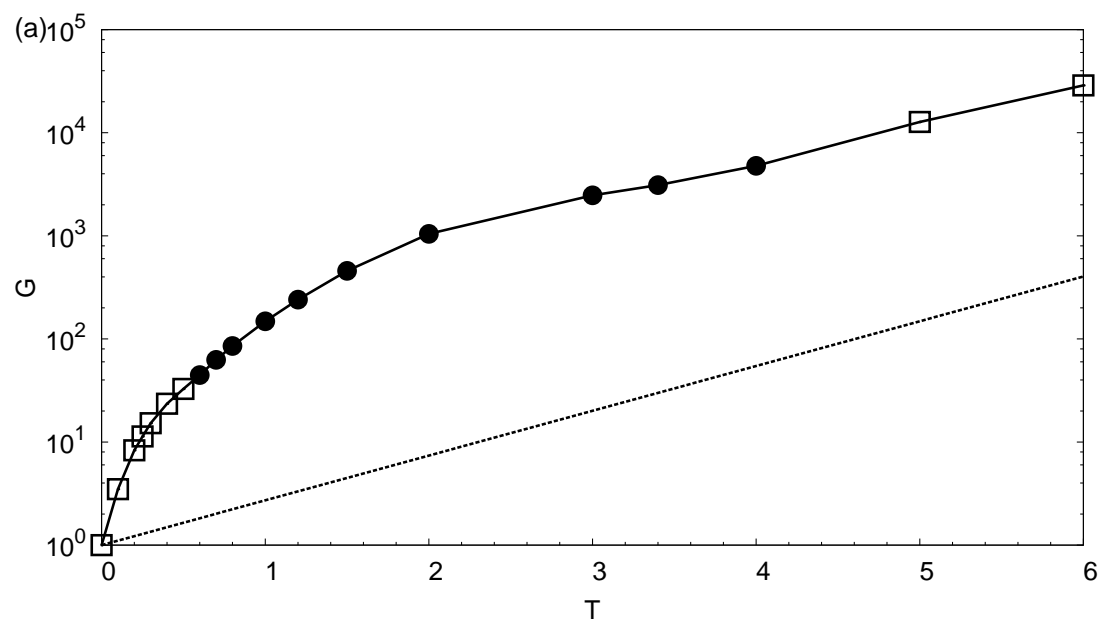

(b)

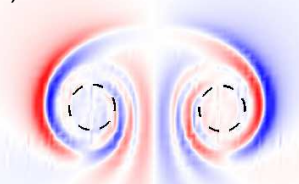

(c)

(d)

FiguRE 18. $R e=1000$ vortex pair: (a) variation of the linear optimal gain with horizon time. The gain obtained using the most unstable mode as the initial perturbation is indicated by a dotted line. The bottom row shows the velocity component parallel to the center-line for the LO-P corresponding to (b) $T=0.5$, (c) $T=1.0$ and, (d) $T=5.0$. The solid circles (resp. hollow squares) in (a) correspond to data points for which the LO-P is symmetric (resp. anti-symmetric).

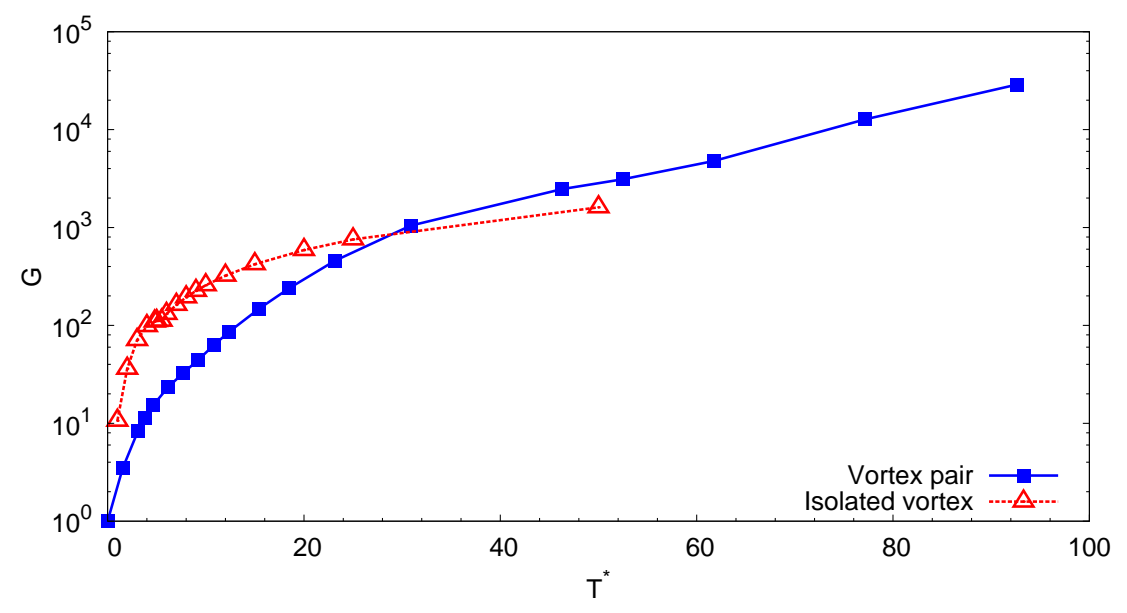

Figure 19. $R e=1000$ vortex pair: Variation of the LO-G with horizon time rendered non-dimensional by the rotation period of the vortex. Also shown is the LO-G curve for an isolated vortex for $R e=5000$. 
(a)
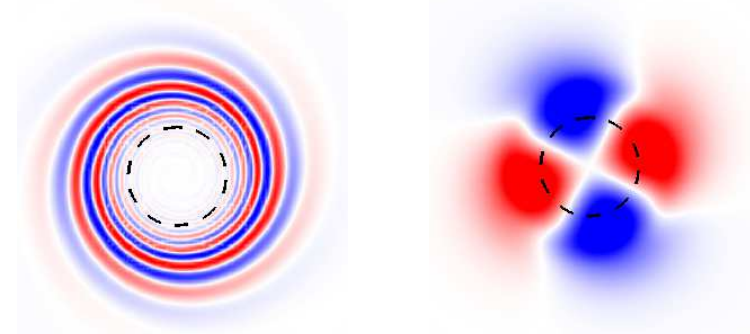

(b)

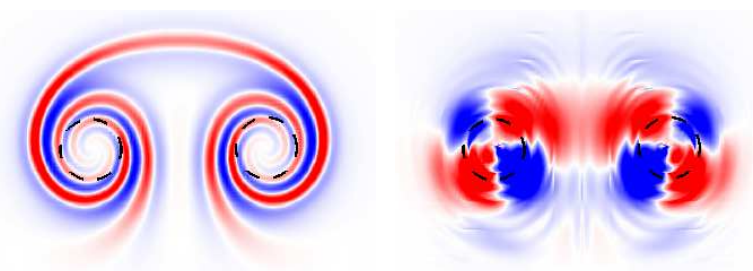

FIGURE 20. Perturbation vorticity field for (a) $R e=5000$ isolated vortex and (b) $R e=1000$ vortex pair. The left column shows the field corresponding to $T^{*} \sim 2.5 \mathrm{LO}-\mathrm{P}$ and the right column shows its evolved state at $t=T^{*}$.

about the center-line. Within the linear framework, the two components evolve independently from each other. Therefore the LO-P can be either symmetric or anti-symmetric. A common approach adopted for optimization of counter-rotating vortex pair, is to use half of the computational domain and prescribe symmetry or anti-symmetry boundary condition on the center-line. In this approach, symmetric and anti-symmetric LO-P are obtained separately. We have used a different approach where computations are carried out for the full computational domain. This is because of the unavailability of antisymmetry boundary condition in Nek5000. LO-P obtained using the full domain approach is the one that has higher energy gain between symmetric and antisymmetric LO-P. For $T<0.6$ and $T>4.0 \mathrm{LO}-\mathrm{P}$ is anti-symmetric, whereas for $0.6 \leqslant T \leqslant 4.0$ the LO-P is symmetric. Figure 18(b-d) gives the velocity component normal to the center-line for the LO-P corresponding to three horizon times. With increasing T, LO-P moves away from the vortex center. For large $T$, LO-P is primarily located on the Kelvin oval and along the center-line.

We now compare LO-G for an isolated vortex and vortex pair. To do so, we chart the variation of the LO-G with $T^{*}$ on log-lin axes where $T^{*}$ is the horizon time rendered non-dimensional by the rotation time of a vortex $\left(T^{*}=T \tau_{b} / \tau\right.$ and $\left.\tau=4 \pi^{2} a^{2} / \Gamma\right)$ in figure 19. The LO-G curve for the isolated vortex presented earlier (see Section 3.2) is included in figure 19. Since, for a vortex pair, $G$ increases exponentially with $T$ for large $T$ (figure 18a), same variation is observed in $G-T^{*}$ curve for large $T^{*}$. In figure 19, the exponential behavior for vortex pair sets in for $T^{*} \gtrsim 60$. For $T^{*} \lesssim 60$, the variation of $G$ with $T^{*}$ for a vortex pair is qualitatively similar to that of isolated vortex. Furthermore, for a given $T^{*}$, the linear evolution of the LO-P for the two systems are also similar. We consider linear flow evolution for $T^{*}=2.5 \mathrm{LO}-\mathrm{P}$ to demonstrate this. The initial perturbation is anti-symmetric about the center-line. Figure 20 shows the shape of LO-P and its evolved state at $T^{*}$. The LO-P for both isolated and vortex-pair system consist of vorticity filaments in a spiral arrangement around the vortex core. The evolved perturbation state at $T^{*}$ has a quadrupolar structure near the vortex center. Therefore it appears that the mechanism for the largest gain in perturbation energy for a vortex 

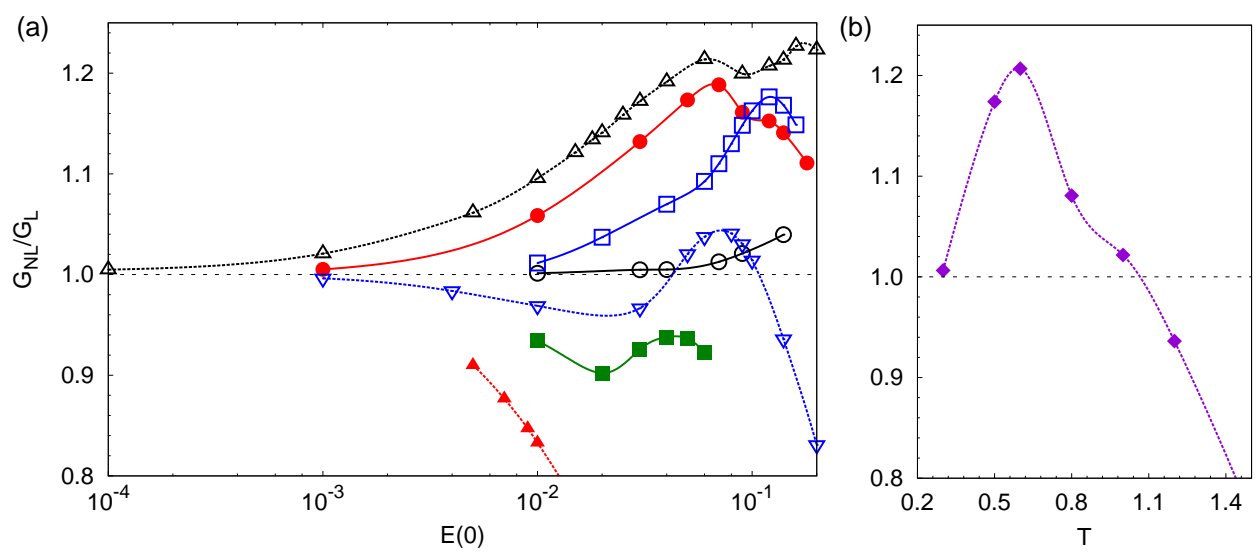

FiguRE 21. $R e=1000$ vortex pair: variation of the nonlinear optimal gain with (a) the initial energy of the perturbation for different horizon times, $T=0.3(\circ), T=0.5(\bullet), T=0.6(\triangle)$, $T=0.8(\square), T=1.0(\nabla), T=1.2(\square)$ and $T=2.0(\boldsymbol{\Delta})$, (b) the horizon time for $E(0)=0.05$.
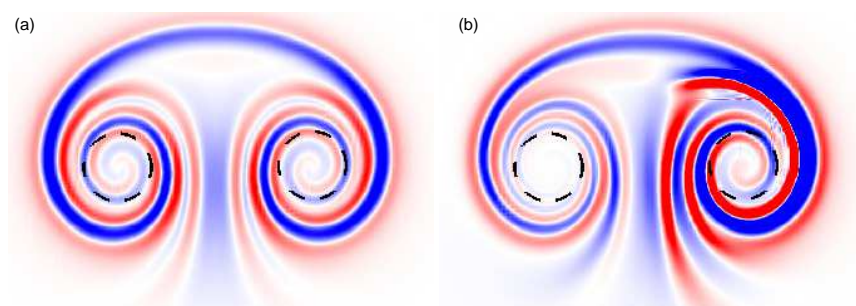

FiguRE 22. $R e=1000$ vortex pair: perturbation vorticity field of the $T=0.6$ nonlinear optimal perturbation for (a) $E(0)=10^{-4}$ and (b) $E(0)=0.02$.

pair is the same as that of an isolated vortex. In other words, the interaction between the two vortices of the vortex pair has no significant effect on the physical processes that yield the largest energy gain. Same inference is made with flow evolution of symmetric LO-P.

\subsection{Nonlinear optimization}

Figure 21 shows the variation of the NLO-G with initial energy of the perturbation $(E(0))$ for various horizon times. NLO-G has been normalized using the LO-G. As for the isolated vortex, there exists a threshold value of $E(0)$ below which NLO-G is nearly the same as the LO-G. Above this threshold, the optimal gain is different from the LOG. With increasing $T$, the threshold $E(0)$ decreases. The peak difference between the nonlinear and linear optimal gains increases with horizon time up to $T=0.6$ (figure $21 \mathrm{~b})$. For $T=0.6$, the NLO-G is higher than the LO-G for the largest range of $E(0)$. For $T>1.0$, the NLO-G is lower than the LO-G for all the values of $E(0)$ that were considered in this work.

Next we discuss the shape of the NLO-P for a vortex pair. Figure 22 shows the vorticity field of the $T=0.6$ nonlinear optimal perturbation for $E(0)=10^{-4}$ and $E(0)=2 \times 10^{-2}$. The threshold energy for $T=0.6$ is $E(0)=6 \times 10^{-4}$. Below the threshold energy, the nonlinear and linear optimal perturbations have identical shape. Above the threshold energy, the nonlinear optimal perturbation has an asymmetric structure: the perturbation is stronger in the positive vortex than the negative vortex. 


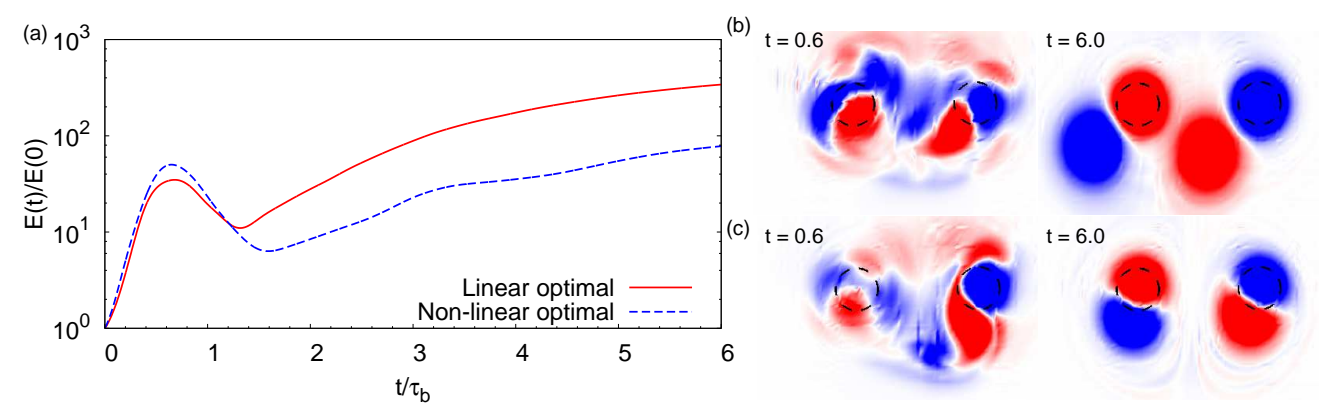

Figure 23. $R e=1000$ vortex pair: (a) time evolution of the energy gain for computations initiated with the LO-P and the $E(0)=0.02$ nonlinear optimal perturbation. (b) Perturbation vorticity field at $t=0.6$ and $t=6.0$ for the computation initiated with the LO-P. (c) Perturbation vorticity field at $t=0.6$ and $t=6.0$ for the computation initiated with the $E(0)=0.02$ nonlinear optimal perturbation.

\subsection{Long-term nonlinear evolution of the optimal perturbation}

Figure 23 gives the time history of the energy gain for computations initiated with the LO-P and the $E(0)=0.02$ NLO-P for $T=0.6$. The initial energy of the LO-P is also set to $E(0)=0.02$. Initially, the perturbation energy increases because of the transient processes. The peak gain reached during the transient phase for NLO-P is higher than that reached by the LO-P. Long-term the LO-P results in higher energy gain than the NLO-P. The perturbation vorticity field at two time instants during the flow evolution are shown in figures $23(\mathrm{~b}, \mathrm{c})$. For the LO-P, the flow field at $t=T$ is nearly symmetric, whereas for the nonlinear optimal the flow field is asymmetric. At large times $(t=6)$, the perturbation has a dipolar structure near the vortex core. The vortex dipoles provoke a displacement of the vortex cores in the left and downwards direction.

\section{Conclusions}

Results have been presented for the optimal perturbation of single and double vortex systems. The optimal perturbation corresponds to the initial condition that results in largest gain of perturbation energy for a given horizon time $T$. A direct-adjoint approach is employed to compute the optimal perturbations and corresponding gains. Both linear and nonlinear optimization have been performed.

The single vortex system is modeled as isolated $2 D$ Lamb-Oseen (LO) vortex. The Reynolds number based on the vortex circulation is $R e=5000$. Linear analysis showed that the optimal perturbation for low horizon time $T$ is of azimuthal wavenumber $m \geqslant 2$. For large $T$ the linear optimal perturbation (LO-P) is of $m=1$. In general, LO-P consists of vorticity filaments in a spiral arrangement. With increasing horizon time $T$, the vorticity filaments move away from the vortex center. For low values of the initial perturbation energy $(E(0))$, linear and nonlinear optimal analysis yield the same result. However, beyond a threshold value of $E(0)$, the nonlinear optimal perturbation (NLO$\mathrm{P}$ ) and the corresponding gain is different from that obtained via linear computation. Fourier decomposition of the NLO-P beyond the threshold energy shows contributions from multiple azimuthal wavenumbers. The interaction between different wavenumbers results in higher optimal gain than that of the linear optimal for a range of $E(0)$.

Long-term nonlinear evolution of linear and nonlinear optimal perturbations is studied. It is observed that after the transient energy growth, the perturbation relaxes towards a quasi-steady non-axisymmetric state. The shape of the perturbation in the quasi-steady 


$\begin{array}{lcc} & \text { Frozen base flow } & \text { Diffusing base flow } \\ \text { Linear } & 109.4 & 109.4 \\ \text { Non-linear } E_{0}=10^{-2} & 140.3 & 139.8\end{array}$

TABLE 2. Linear and nonlinear $E_{0}=10^{-2}$ optimal gains $G(T)$ obtained for a Lamb-Oseen vortex at $R e=5000$, with $T=4.8$ using a frozen base flow and a diffusing base flow.

state depends on the structure of the optimal perturbation. For example, for the $T=4.8$ linear optimal perturbation the quasi-steady state consists of six vorticity patches, while for the $T=4.8$ NLO-P, the quasi-steady state has a tripolar structure. In the tripolar structure, a satellite vortex rotates around a vortex dipole. The appearance of the satellite vortex is attributed to a nonlinear critical layer effect. The optimal perturbations, linear and nonlinear, are a good initial condition to obtain non-axisymmetric vortices.

The two-vortex system has been modeled as a pair of equal strength counter-rotating vortices. Such a flow configuration is relevant for the study of vortex dynamics in the wake of an aircraft. A counter-rotating vortex pair is a linearly unstable flow system (Brion et al. 2014). However it supports transient growth of perturbation energy that may lead to significantly higher energy gain than that reached by the most unstable mode. For small and large horizon times $T$, the LO-P of vortex pair is anti-symmetric about the center-line, whereas for moderate $T$, the LO-P is symmetric. It is observed that for $2 D$ perturbations, the mechanism of transient growth in a vortex pair is similar to that of an isolated vortex. Adding a counter-rotating vortex in the vicinity of a first vortex does not have a significant effect on the shape of the optimal perturbation within the first vortex. Similarly to the isolated vortex, a threshold value of $E(0)$ exists beyond which the nonlinear optimal gain is higher than the linear optimal gain. In this situation the NLO-P is asymmetric about the center-line.

\section{Acknowledgment}

We are grateful to Dr. Mirko Farano, DMMM, Politecnico di Bari, Italy for his help in developing the numerical optimization tool. We would also like to thank Prof. Pierre Brancher, IMFT, Toulouse for his support and sharing Luigi Bisanti's experience on nonlinear optimization. This work has been supported by the French Ministry of Civil Aviation (DGAC) under the PHYWAKE (Physics of Wake Vortices) research program. We thank the anonymous referees for their valuable feedback and insightful suggestions.

\section{Appendix A. Validation of the frozen base flow approach}

In this appendix, the validity of frozen base flow approach for linear and nonlinear optimization process is demonstrated. We select $T=4.8$ and compute the nonlinear and linear optimal gain with frozen as well as diffusing base flow. Table 2 lists the values of the optimal gain obtained via the two approaches. It can be observed that the difference between the two approaches is less than $0.5 \%$. The slightly lower value of optimal gain obtained using diffusing base flow is attributed to reduction of vortex strength under the action of viscosity. 


$\begin{array}{lc}T & C_{\text {mum }}(T) \\ 0.1 & 0.7 \\ 0.25 & 1.9 \\ 0.4 & 3.5 \\ 4.0 & 39.2 \\ 6.0 & 41.6\end{array}$

TABLE 3 . Value of $C_{\text {mum }}$ (defined by B 1) calculated for LO-P corresponding to various horizon times.

\section{Appendix B. Vortex pair: contribution of most unstable mode to LO-P}

A pair of counter-rotating planar vortices is a linearly unstable flow system (Brion et al. 2014). The long time response of the linearized system will be governed by the most unstable mode. However, owing to the non-normal nature of the linearized operator, the most unstable mode alone cannot be representative of short time transient flow processes. To confirm this, we calculate the following ratio for LO-P computed for various horizon times:

$$
C_{m u m}(T)=\frac{\left(\mathbf{u}_{\mathbf{o p t}}(\mathbf{T}), \mathbf{u}_{\mathbf{m}}^{+}\right)}{\left(\mathbf{u}_{\mathbf{m}}, \mathbf{u}_{\mathbf{m}}^{+}\right)} .
$$

In (B 1), the numerator on the right hand side is the scalar product between the velocity field corresponding to linear optimal perturbation $\left(\mathbf{u}_{\mathbf{o p t}}\right)$, and adjoint of the most unstable mode of the vortex-pair system $\left(\mathbf{u}_{\mathbf{m}}^{+}\right)$. The denominator on the right hand side of (B 1) is the scalar product between the most unstable mode $\left(\mathbf{u}_{\mathbf{m}}\right)$ and $\mathbf{u}_{\mathbf{m}}^{+}$. In the calculation of the scalar product, only spatial integration is carried out (unlike the inner product defined in (2.9) where the integration is carried out in both space and time), and all the vectors are normalized with respect to their norm. The ratio $C_{m u m}$ is a measure of the contribution of the most unstable mode (mum) to LO-P. This follows from the biorthogonality relation between the eigenmodes of the direct and adjoint system. Table 3 shows the value of $C_{m u m}$ calculated for LO-P corresponding to various horizon times. For large $T, C_{\text {mum }}$ is relatively high. This suggests large contribution of the most unstable mode in long time LO-P. On the other hand, for low $T$ the contribution of most unstable mode to LO-P is low as is evidenced by relatively small value of $C_{\text {mum }}$.

\section{REFERENCES}

Antkowiak, A. \& Brancher, P. 2004 Transient energy growth for the Lamb-Oseen vortex. Physics of Fluids 16 (1), L1-L4.

Antkowiak, A. \& Brancher, P. 2007 On vortex rings around vortices: an optimal mechanism. Journal of Fluid Mechanics 578, 295-304.

BARKLEY, D. 2016 Theoretical perspective on the route to turbulence in a pipe. Journal of Fluid Mechanics 803.

Bernoff, A. J. \& Lingevitch, J. F. 1994 Rapid relaxation of an axisymmteric vortex. Physics of Fluids 6 (11), 3717-3723.

BisAnti, L. 2013 Linear and nonlinear optimal perturbation analysis of vortices in incompressible flows. PhD Thesis.

Brion, V. 2009 Stabilité de paires de tourbillons contra-rotatifs: application au tourbillon de jeu dans les turbomachines. PhD Thesis. 
Brion, V., Sipp, D. \& JACquin, L. 2014 Linear dynamics of the Lamb-Chaplygin dipole in the two-dimensional limit. Physics of Fluids 26 (6), 064103.

Cherubini, S., De Palma, P., Robinet, J,-C. \& Bottaro, A. 2011 The minimal seed of turbulent transition in the boundary layer. Journal of Fluid Mechanics 689, 221-253.

Cherubini, S. \& De Palma, P. 2013 Nonlinear optimal perturbations in a Couette flow: bursting and transition. Journal of Fluid Mechanics 716, 251-279.

Corbett, P. \& Bottaro, A. 2000 Optimal perturbations for bondary layers subject to streamwise pressure gradient. Physics of Fluids 12, 120-130.

Crow, S. C. 1970 Stability theory for a pair of trailing vortices. AIAA journal 8 (12), $2172-$ 2179.

Douglas, S. C., Amari, S.-I. \& Kung, S.-Y. 2000 On gradient adaptation with unit-norm constraints. IEEE Transactions on Signal Processing 48 (6), 1843-1847.

Fabre, D., Sipp, D. \& Jacquin, L. 2006 Kelvin waves and the singular modes of the LambOseen vortex. Journal of Fluid Mechanics 551, 235-274.

FARrell, B. F. 1988 Optimal excitation of perturbations in viscous shear flow. Physics of Fluids 31 (8), 2093.

Fischer, P., Lottes, J. \& Kerkemeier, S. 2008 Nek5000 Web Page. http://nek5000.mcs.anl.gov.

Green, S. I. 1995 Fluid vortices. Dordrecht: Kluwer Academic Publishers.

Habermann, R. 1972 Critical Layers in Parallel Flows. Studies in Applied Mathematics 51 (2), $139-161$.

Jugier, R. 2016 Stabilité bidimensionnelle de modeles de sillage d'aéronefs. PhD Thesis.

KeLvin, LORD 1880 Vibrations of a columnar vortex. The London, Edinburgh, and Dublin Philosophical Magazine and Journal of Science 10 (61), 155-168.

Le Dizes, S. 2000 Non-axisymmetric vortices in two-dimensional flows. Journal of Fluid Mechanics 406, 175-198.

Kerswell, R. R. 2018 Nonlinear nonmodal stability theory. Annual Review of Fluid Mechanics $50(1)$.

Le Dizes, S. 2000 Non-axisymmetric vortices in two-dimensional flows. Journal of Fluid Mechanics 406, 175-198.

Lugt, H. J. 1983 Vortex flow in nature and technology. New York: Wiley.

MaO, X. \& Sherwin, S. 2011 Continuous spectra of the Batchelor vortex. Journal of Fluid Mechanics 681, 1-23.

Mao, X. \& Sherwin, S. 2012 Transient growth associated with continuous spectra of the Batchelor vortex. Journal of Fluid Mechanics 697, 35-59.

OrR, W. McF. 1907 Stability or instability of the steady motions of a perfect liquid. Proc. Ir. Acad. Sect. A, Math Astron. Phys. Sci 27, 9-69.

Pierrehumbert, R. 1980 A family of steady, translating vortex pairs with distributed vorticity. Journal of Fluid Mechanics 99 (1), 129-144.

Pradeep, D. S., \& Hussain, F. 2006 Transient growth of perturbations in a vortex column. Journal of Fluid Mechanics 550, 251-288.

Rossi, L. F., Lingevitch, J. F. \& Bernoff, A. J. 1997 Quasi-steady monopole and tripole attractors for relaxing vortices. Physics of Fluids 9 (8), 2329-2338.

Saffman, P. G. 1992 Vortex dynamics. Cambridge: Cambridge University Press.

Schmid, P. J. \& BRAndt, L. 2014 Analysis of fluid systems: Stability, receptivity, sensitivitylecture notes from the FLOW-NORDITA summer school on advanced instability methods for complex flows, Stockholm, Sweden, 2013. Applied Mechanics Reviews 66 (2), 024803.

Sipp, D., JACQuin, L. \& Cossu, C. 2000 Self-adaptation and viscous selection in concentrated two-dimensional vortex dipoles. Physics of Fluids 12 (2), 245-248.

Trefethen, L., Trefethen, A., Reddy, S. \& Driscoll, T. 1993 Hydrodynamic stability without eigenvalues. Science 261 (5121), 578-584.

Tsai, C.-Y., \& Widnall, S. E. 1976 The stability of short waves on a straight vortex filament in a weak externally imposed strain field. Journal of Fluid Mechanics 73 (04), 721-733.

Zuccher, S., Bottaro, A. \& Luchini, P. 2006 Algebraic growth in a Blasius boundary layer: Nonlinear optimal diturbances. European Journal of Mechanics-B/Fluids 25, 1-17. 